\title{
Not Only Barbara
}

\author{
Paul J. E. Dekker
}

Published online: 22 February 2015

(c) The Author(s) 2015. This article is published with open access at Springerlink.com

\begin{abstract}
With this paper I aim to demonstrate that a look beyond the Aristotelian square of opposition, and a related non-conservative view on logical determiners, contributes to both the understanding of Aristotelian syllogistics as well as to the study of quantificational structures in natural language.
\end{abstract}

Keywords Categorial logic - Square of opposition - Cube of oppositions . Generalized quantifiers - Conservativity - Topical restriction - Focus sensitivity . Existential there Only $\cdot$ Many

\section{Introduction}

Aristotelian syllogistics is confined to a square of oppositions that has fascinated linguists and logicians for centuries. It has overshadowed a cube of oppositions, discovered by Reichenbach some decades ago, of which the square constitutes just one side. In this paper I restage this cube and uncover its syllogistics, polishing and slightly improving Reichenbach's observations (Sect. 2). The one-sided focus on the square has aligned with a bias for conservative determiners in the theory of generalized quantifiers. However, certain structural features of quantificational structures in natural language can be properly delineated only if non-conservative determiners are not excluded from the outset (Sect. 3). I conclude with an outline of some natural and interesting subsections of the cube other than the square (Sect. 4). In each of the three sections the trivial conclusion is that a restricted focus hampers seeing things.

P. J. E. Dekker $(\bowtie)$

ILLC/Department of Philosophy, University of Amsterdam, Science Park 107, 1098 XG Amsterdam, The Netherlands e-mail: p.j.e.dekker@uva.nl 


\section{Categorial Logic}

\subsection{The Square of Opposition}

Aristotle's logic is primarily concerned with the predication of a term $P$ to a subject term $S$ that results as a logical consequence from two independent propositions, relating the two terms to a middle term $M$. Aristotle acknowledged propositions

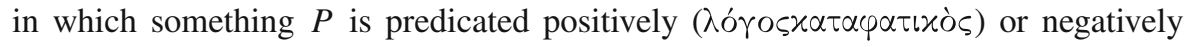

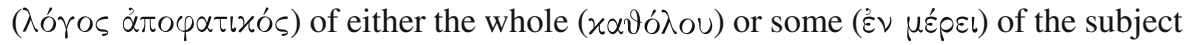
term $S$. Thus, for any two terms $A$ and $B$, there are four types of propositions. There are two general propositions, that all $A$ are $B$ (also rendered as $A a B$ ) and that all $A$ are not $B$, or, equivalently, that no $A$ are $B$ (rendered as $A e B$ ). Two particular propositions state that some $A$ is or are $B(A i B)$ and that some $A$ is not $B$, or, equivalently, that not all $A$ are $B(A o B)$. A categorial proposition thus consists of two general terms which are joined by one of the combinators $a, e, i$, or $o$.

A syllogism concludes to some proposition $S w P$, with $P$ the predicate or 'major' term of the syllogism, $S$ the subject, or 'minor' term, and $w$ one of the four combinators, on the basis of two independent premises. The first (major) premise relates the major term $P$ to a middle term $M$ by means of a combinator $u$, and the second (minor) premise relates the subject term $S$ to the middle term $M$ by means of a combinator $v$. Since the middle term $M$ can figure as both subject and predicate in the premises, a syllogism with the combinators $u, v$ and $w$, in that order, can be of one of the following four forms, also known as the four figures.

(1) \begin{tabular}{cccc}
$M u P$ & $P u M$ & $M u P$ & $P u M$ \\
$\frac{S v M}{S w P}$ & $\frac{S v M}{S w P}$ & $\frac{M v S}{S w P}$ & $\frac{M v S}{S w P}$ \\
\cline { 2 - 2 } & $u v w-2$ & $u v w-3$ & uvw-4
\end{tabular}

A syllogism counts as valid if and only if the conclusion logically follows from the premises..$^{1}$ That is to say, if and only if the relations between predicate and middle term and between middle term and subject that are stated by the premises, logically enforce a specific relation between the predicate and subject themselves, as stated by the conclusion. A clear, also called 'perfect', example is one in which the premises predicate $P$ of all that is $M$, and $M$ of all that is $S$, in which case we can conclude that, if the premises hold, then $P$ applies to all that is $S$. Formally, MaP, $S a M / S a P$. This is a valid syllogism of the first figure, also known as 'Barbara'.

Historically — categorial logic predates propositional Boolean reasoning and predicate logic — syllogistic validity has been taught and studied from a more or less prooftheoretic angle. Without further assumptions 15 syllogistic schemes have qualified as valid. These have been seen to be reducible, by means of suitable transformation rules, to four valid schemes of the first figure. These four have been given mnemonic names later, with vowels reminiscent of their form. (The valid schemes of the other three figures have been baptized analogously.)

\footnotetext{
${ }^{1}$ In the terminology adopted here the term 'syllogism' is used for inferences of a particular form, including invalid ones. This is perhaps not the standard locution, but convenient.
} 
(2) $C a B, A a C / A a B$ (aaa-1: 'Barbara');

$C e B, A a C / A e B$ (eae-1: 'Celarent');

$C a B, A i C / A i B$ (aii-1: 'Darii');

CeB, $A i C / A o B$ (eio-1: 'Ferio').

For some good, but arguably inessential, reasons Aristotle deemed these schemes

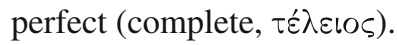

Aristotle's syllogistics can be, and has been, substantiated and supplemented in various ways. The terms $A, B, \ldots$, can be supposed to make a distinction in some ontological (e.g., set-theoretical) space, to the effect that $A$ and not- $A$ (indicated by $A^{\prime}$ ) satisfy at least two conditions: non-contradiction (nothing is both $A$ and $A^{\prime}$ ) and excluded middle (everything is either $A$ or $A^{\prime}$ ). (It is natural to also assume nontriviality, that there actually is or are $A$ and $A^{\prime}$.)

A categorial proposition of the form $A u B$, with $u$ one of the combinators, is intuitively construed as having the $A$ as its subject matter, and what matters is the distinction that $B$ makes among them. The propositions relate to the distinction between the objects that are both $A$ and $B$, and those that are $A$ but not $B$. The general propositions state that there is none of the first kind of objects $(A a B)$ or that there is none of the second kind $(A e B)$. The particular propositions state the opposite, that there is some of the first kind of objects $(A i B)$ or of the second kind $(A o B)$. Assuming that $I(A)$ gives us the extension of $A$ in some universe model or universe $E$ (e.g., $I(A) \subseteq E$ ), the four types of propositions can be given the following set-theoretic interpretation.

(3) $A e B$ (is true relative to $I$ )

$A a B$

$A i B$

iff $I(A) \cap I(B)=\emptyset$;

iff $I(A) \cap \overline{I(B)}=\emptyset$;

iff $I(A) \cap I(B) \neq \varnothing$;

$A o B$

iff $I(A) \cap \overline{I(B)} \neq \varnothing$.

The categorial propositions can be diagrammatically represented in figures in which two circles labeled $A$ and $B$ characterize the theoretically possible interpretations of $A$ and $B$, displaying the possibility of stuff that is $A$ but not $B$, of stuff that is $A$ and $B$, of stuff that is not $A$ but $B$, and of stuff that is neither. To indicate that there is stuff of one of these kinds we can put a cross in the associated area, and to indicate there is none the area is wiped out. Thus, the four propositions can be displayed as follows.
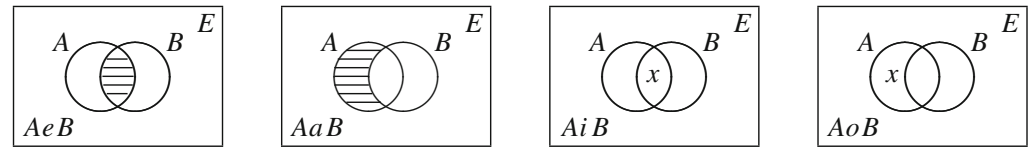

Upon the sketched conception of categorial propositions, the validity of syllogistic schemes can be established by elementary set-theoretic means, and it can be illustrated by means of a diagrammatical method. A syllogism is valid if, and only if, once one has displayed the contribution of two premises in a diagram, the conclusion 'already stands there.' Consider, for instance, the syllogistic scheme Ferio (eio-1). The following diagram portrays the contribution of the major, $C e B$ (the area where $C$ and $B$ overlap is wiped out), and the minor $A i C$ (a cross in both $A$ and $C$ ). 


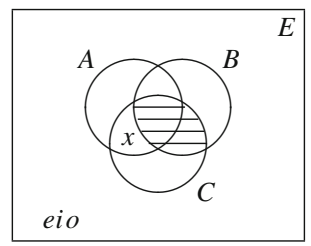

We see that the conclusion $A o B$ of Ferio, indicated by the cross in $A$ but not in $B$, has already been established through the premises. The inference, thus, is valid. The (in-)validity of the other syllogistic schemes can be demonstrated likewise.

Like I said, Aristotle's logic accommodates four types of propositions, which constitute the famous square of oppositions. ${ }^{2}$

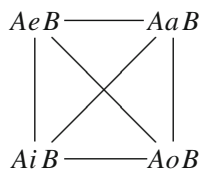

The two vertical lines in this square connect propositions which contradict each other. If we assume non-triviality, the diagonals indicate that the propositions on the top each presuppose the diagonally connected ones on the bottom. The two top propositions, on this assumption, can therefore not both be true, and are called 'contraries'; the two bottom propositions cannot both be false then, and are called 'subcontraries'. Thus, as Aristotle held, "All men are just." and "No man is just." are contraries, since the predicate "just" is both ascribed to and withheld from the substance "man". Assuming, as Aristotle did, that there are instances of "man", individual men, these sentences cannot both be true. Likewise, "Some men are just." and "Not all men are just." cannot both be false then. Upon the stated assumption there must be some man, and he must be either just $(A i B)$ or unjust $(A o B)$.

Standard categorial logic has been confined to the (propositions or combinators from this) square. It is no exaggeration to say that for some it constitutes a wholly logical space. Logically speaking, however, the square fills only part of logical space, as, among few others, Hans Reichenbach observed.

\subsection{Cubing the Square}

In his De Interpretatione, Aristotle already alluded to the possibility to speak, categorially, of things that are not of a certain kind, mentioning propositions made with a

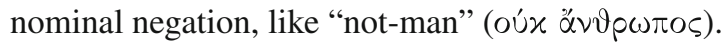

'Every not-man is just' does not signify the same as any of the above, nor does its opposite, 'not every not-man is just'. But 'every not-man is not-just' signifies the same as 'no not-man is just'. (Aristotle 1984, 20³7-9)

\footnotetext{
2 The square is here presented in a slightly unusual order. The presentation is logically speaking isomorphic to the usual one, but more convenient for what follows.
} 
Aristotle here mentions three of four other logically possible categorial propositions. "Every not- $A$ is $B$." ("Unless something is $A$, it is $B$. ."), "Not every not- $A$ is $B$." ("Something is neither $A$ nor $B$. .), and "Every not- $A$ is not- $B$. ." ("Only $A$ are $B$. .) We may, for the sake of completeness, add "Not every not- $A$ is not- $B$." ("Not only $A$ are $B$.") Each of the original four combinators may thus yield a duplicate, which involves the negation of its subject term, and we will use four new combinators $(\ddot{a}, \ddot{o}$, $x$ and $y$ ) to express precisely these duplicates. (Here, together with a reformulation, slightly unhappy if in lack of a better alternative.)

(4) $A \ddot{a} B$ equivalent with $A^{\prime} e B \quad($ Only $A$ are $B)$;
$A x B$
$A^{\prime} a B \quad$ (Unless $A$ (it is) $B$ );
$A \ddot{o} B$
$A^{\prime} i B \quad($ Not only $A$ are $B)$;
$A y B$
$A^{\prime} o B \quad($ Else $A B$, something is neither $A$ nor $B)$.

The four new combinators have already been identified and studied by Augustus de Morgan and Charles Sanders Peirce, among others (de Morgan 1846; Peirce 1931, 1880). The findings of these studies have, however, not become widespread, presumably because of the decline of categorial logic in the 19th century, together with the rise of more general propositional and predicate logics - of course, partly thanks to the very same authors themselves. The interpretation of the new combinators is obvious.

(5) $\begin{array}{ll}A \ddot{a} B \text { (is true) } & \text { iff } \overline{I(A)} \cap I(B)=\emptyset ; \\ A x B & \text { iff } \overline{I(A)} \cap \overline{I(B)}=\emptyset ; \\ A \ddot{o} B & \text { iff } \overline{I(A)} \cap I(B) \neq \emptyset ; \\ A y B & \text { iff } \overline{I(A)} \cap \overline{I(B)} \neq \emptyset .\end{array}$

The propositions $A \ddot{a} B$ and $A \ddot{o} B$ turn out to be the converses of $A a B$ and $A o B$, since they state that $B a A$ and $B o A$, respectively. ${ }^{3}$ The $x$ - and $y$-propositions go beyond the expressive power of the Aristotelian square. The proposition $A x B$ states that $A$ and $B$ are exhaustive, to the effect that everything is (at least) $A$ or $B$, and $A y B$ says that there exists something beyond the two terms. ${ }^{4}$ It is easily seen that the four new combinators complete the set of minimal logical possibilities invited by the diagrammetrical representation of the categorial propositions.
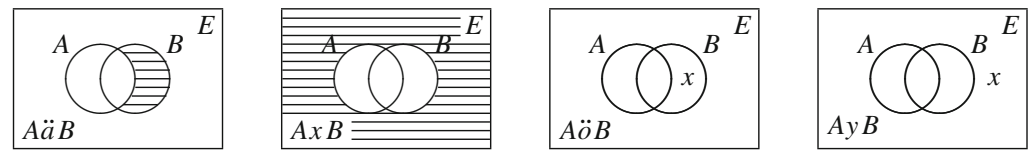

The new set of combinators naturally makes up another square of oppositions.

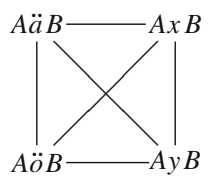

\footnotetext{
3 This is why I have chosen to use the 'umlaut'-ed vowels, like (Kraszewski 1956).

4 Running out of vowels, I have chosen ' $y$ ', because it most looks like one, and ' $x$ ', mnemonic for $e x$-haust.
} 
This is not surprising of course, because the new square of opposition is the inverse of the original one. Define the inverse $(A u B)^{i}$ of a proposition $A u B$ as $A u^{i} B=A^{\prime} u B^{\prime}$. We then find that:

(6)

$$
\begin{array}{ll}
(A e B)^{i}=A x B & (A a B)^{i}=A \ddot{a} B \\
(A i B)^{i}=A y B & (A o B)^{i}=A \ddot{o} B \text { and vice versa. }
\end{array}
$$

The syllogistics of the inverted square of course mirrors that of the square.

(7) A syllogistic scheme $u v w-x$ is valid iff its inverse $u^{i} v^{i} w^{i}-x$ is. ${ }^{5}$

Logically speaking, the new square thus does not give us anything new, all by itself. However, new results are obtained from the interaction of the two squares.

Reichenbach (1952) has joined the two squares in a logical cube of oppositions. ${ }^{6}$ In the following picture the traditional square figures as the front-if it pleases one to conceive the cube that way, and the new, inverted, square as the back-side.

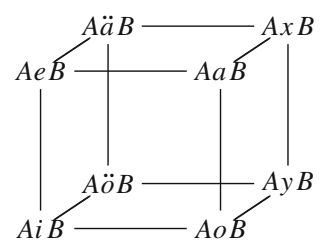

As before, vertical lines relate contradictory propositions. The horizontal lines indicate predicate negation, and the lines 'from back to front' indicate subject negation. More logical structure can be observed in the cube if we assume the propositions not to be 'degenerate', as Hans Reichenbach calls it. A proposition $A u B$ is not degenerate if the terms $A$ and $B$ make non-trivial, independent distinctions. Together with the nontriviality condition mentioned above, associated with a proper use of the terms, this leads to the assumption that a proposition comes with a presupposition of 'differential import', as we prefer to call it.

(8) The differential import of a proposition $A u B$ is that $A$ and $B$ make a different proper distinction, i.e., $A \neq \emptyset \neq A^{\prime}, B \neq \emptyset \neq B^{\prime}$ and $A \neq B \neq A^{\prime}$.

Reichenbach observed that, on the assumption of differential import, all corners of the cube stand in logical relations. The assumption makes any one of the top propositions (the four general ones) presuppose the three bottom propositions (particular ones) that they do not contradict. Hence, all four top propositions are contraries, because no two of them can be true together. For the same reason all four bottom propositions are subcontraries, since no two of them can be false together.

The cube is logically convenient, because it is both negation- and conversecomplete. This is to say that every proposition $A u B$ has a unique negation $A u^{\prime} B=$ $(A u B)^{\prime}$, a unique predicate negation $(A u B)^{P^{\prime}}=A u B^{\prime}$, and a unique subject negation $(A u B)^{S^{\prime}}=A^{\prime} u B$. Also, every proposition $A u B$ has a (unique) converse

\footnotetext{
5 Obviously, $u^{i} v^{i} w^{i}$ is schematically the same as $u v w$ except for the fact that each of the terms $P, S$ and $M$ have been systematically replaced by $P^{\prime}, S^{\prime}$ and $M^{\prime}$, respectively.

6 Janusz Ciuciura (p.c.) pointed out to me that the Polish logician Tadeusz Czežowski has identified this cube already in 1931. His work has however been inaccessible to me.
} 
$(A u B)^{c}=B v A$ equivalent with $A u B$. The equivalences are summarized in the following table.

\begin{tabular}{|c|cccc|}
\hline $\begin{array}{c}\text { Conn. } \\
A u B\end{array}$ & $\begin{array}{c}\text { Conv. } \\
(A u B)^{c}\end{array}$ & $\begin{array}{c}\text { Neg. } \\
(A u B)^{\prime}\end{array}$ & $\begin{array}{c}\text { P-Neg. } \\
(A u B)^{P^{\prime}}\end{array}$ & $\begin{array}{c}\text { S-Neg. } \\
(A u B)^{S^{\prime}}\end{array}$ \\
\hline$A e B$ & $B e A$ & $A i B$ & $A a B$ & $A \ddot{a} B$ \\
$A a B$ & $B \ddot{a} A$ & $A o B$ & $A e B$ & $A x B$ \\
$A i B$ & $B i A$ & $A e B$ & $A o B$ & $A \ddot{o} B$ \\
$A o B$ & $B \ddot{o} A$ & $A a B$ & $A i B$ & $A y B$ \\
$A \ddot{a} B$ & $B a A$ & $A \ddot{o} B$ & $A x B$ & $A e B$ \\
$A x B$ & $B x A$ & $A y B$ & $A \ddot{a} B$ & $A a B$ \\
$A \ddot{o} B$ & $B o A$ & $A \ddot{a} B$ & $A y B$ & $A i B$ \\
$A y B$ & $B y A$ & $A x B$ & $A \ddot{o} B$ & $A o B$ \\
\hline
\end{tabular}

It can be seen, fairly trivially, that every proposition $A u B$ can be expressed using the combinator $a$ only, with suitable negations, or, for that matter, using $e$, or $x$, or any of the other combinators, together with suitable negations. These negations also obviously shift the polarities of the arguments of the eight combinators. These polarities indicate the 'monotonic' entailment patterns the combinators give rise to, and will be obvious. For instance, both arguments of the combinator $i$ are positive, which can be written as $+i+$, and those of its negation $e$ are both negative, written as $-e$-. The eight combinators can therefore be displayed in a cube of polarities.

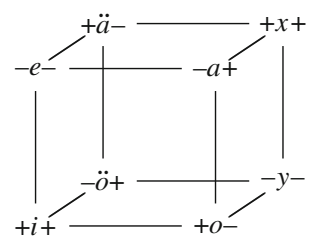

\subsection{Cubistic Syllogistics}

Since the cube is converse- and negation-complete, its logic is quite transparent. Conversions help us get around some practical limitations imposed by the standard presentation of the syllogisms. As said, upon its standard presentation the first, major, premise of a syllogism should contain the major term $P$ of the conclusion, and the minor should contain the subject term $S$. But, of course, it is immaterial, logically speaking, in what order the arguments of a valid inference are presented. If a proposition $r$ follows from propositions $p$ and $q$, it follows from $q$ and $p$. The slightly annoying point is that if we reorder the premises of an inference of the first figure, the subject and predicate terms switch roles, and the middle term switches places in both of the premises. But since we found the cube conversion complete, we can rescue the situation. The role switch of the subject and the predicate term can be made undone by a, meaning preserving, conversion on the conclusion, and the double switch of the middle term can be made undone by a conversion of the two premises.

\section{Observation 1 (Reorder)}

- A syllogistic scheme uvw-1 is valid iff $v^{c} u^{c} w^{c}-1$ is valid. 
(If one scheme is valid if and only if another scheme is, we henceforth call the two schemes 'equivalid'.) Since all combinators in the cube have their converses, re-ordering of premises is always possible.

Conversions also help crossing the borders between the four figures. By a conversion of its premises a syllogism in one figure can be translated into an equivalid one in another figure. If we have a syllogism $B u C, A v B / A w C$ of the first figure, and we use $(B u C)^{c}$, which is of the form $C u^{c} B$, instead of $B u C$, we get a syllogism of the second figure, and since $B u C$ and $C u^{c} B$ are equivalent, the latter syllogism is valid if and only if the first is. This insight gives us a recipe to quadruply any valid syllogism.

\section{Observation 2 (Conversion)}

- The syllogistic schemes $u v w-1, u^{c} v w-2, u v^{c} w-3$ and $u^{c} v^{c} w-4$ are equivalid.

The two observations also tell us how to reorder premises in the figures other than the first one. For, also, $u v w-2$ and $v u w^{c}-2$ are equivalid, as are $u v w-3$ and $v u w^{c}-3$, as well as $u v w-4$ and $v^{c} u^{c} w^{c}-4$.

The two observations above directly entail that, of course, a syllogistic scheme like $e i o$-i is valid, if in one figure, then in all figures, because its premises are selfconverses. As one might have suspected, we also find that the classical syllogistic schemes Celarent (eae-1), Cesare (eae-2), Camestres (aee-2) and Camenes (aee-4) are conversions and reorderings of each other, including, e.g., the scheme dubbed Cälerent (äee-1) here.

\begin{tabular}{|c|c|c|c|}
\hline$M e P$ & & PeM (conv.) & SaM (reord.) \\
\hline SaM & & SaM & PeM \\
\hline$\overline{\mathrm{SeP}}$ & (Celarent) & $\overline{\mathrm{SeP}}$ (Cesare) & $\overline{P e S}$ (Camestres) \\
\hline$M \ddot{a} S$ & (reord.) & SaM (conv.) & \\
\hline$P e M$ & & $M e P$ & \\
\hline$\overline{P e S}$ & (Cälerent) & $\overline{P e S}$ (Camenes) & \\
\hline
\end{tabular}

Other classical schemes like Darii (aai-1), Datisi (aii-3), Disamis (iai-3) and Dimaris (iai-4) are also conversions and reorderings of each other. It can also be seen that the traditional schemes Barocco (aoo-2) and Bocardo (oao-3) have no equivalent conversion into the first figure, if we only have the combinators from the square. With the combinators from the cube we have one, for instance, $\ddot{a} \circ o-1$, (Bärboro). This scheme can be illustrated by means of the following (valid) syllogism.

(9) Only mentalists are Platonist. (MäP)

Not all solipsists are mentalist. (SoM)

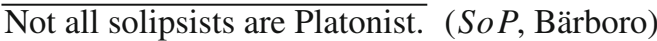

The Aristotelian square also does not enable the conversion of Barbara (aaa-1) into another figure. With the cube, however, Barbara can be converted, and reordered. Reordering Barbara gives us, naturally tagged, Bärbärä ( $\ddot{a} \ddot{a} \ddot{-1})$. (Which is also its inversion, by the way.)

$\begin{array}{ll}M a P & M \ddot{a} S \text { (reord.) } \\ \frac{S a M}{S a P} \text { (Barbara) } & \frac{P \ddot{a} M}{P \ddot{a} S} \text { (Bärbärä) }\end{array}$


The two observations, hence, actually tell us that the logic of the cube, contrary to that of the square, is not constrained by the geometry of the four figures.

Since the cube is negation-complete, and the syllogistic validities are schematic, we can obtain valid syllogistic schemes from other valid schemes by systematically negating the predicate term, the subject term, the middle term, or any number of them. This is laid down in the following, fairly obvious, observation about syllogisms of the first figure.

Observation 3 (Negation) A syllogistic scheme uvw-1 is valid iff $u^{P^{\prime}} v w^{P^{\prime}}-1$ is valid (P-Negation) iff $u v^{S^{\prime}} w^{S^{\prime}}-1$ is valid (S-Negation) iff $u^{S^{\prime}} v^{P^{\prime}} w-1$ is valid (M-Negation).

For example, the validity of Barbara (aaa-1) implies that of Celarent (eae-1).

$$
\begin{aligned}
& \text { MaP hence } P^{\prime} \quad M a P^{\prime} \text { which is } M e P \\
& \frac{S a M}{S a P} \text { (Barbara) } \frac{S a M}{S a P^{\prime}} \quad \frac{S a M}{S e P} \text { (Celarent) }
\end{aligned}
$$

By the negation of the subject and the middle term also the schemes baptized Gaxx

\begin{tabular}{|c|c|c|}
\hline$M a P$ hence $S^{S^{\prime}}$ & $M a P$ which is & $M a P$ \\
\hline SaM & $S^{\prime} a M$ & $S x M$ \\
\hline$\overline{S a P}$ (Barbara) & $\overline{S^{\prime} a P}$ & $\overline{S x P}$ (Gaxx) \\
\hline
\end{tabular}
$(a x x-1)$ and Xena (xea-1) show up valid.

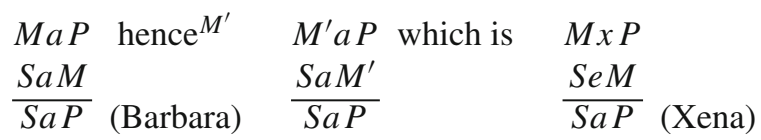

The last scheme can be illustrated by the following line of (sound) reasoning.

(10) Everybody was on a holiday or at a conference. $(H x C)$

Nobody who replied was on a holiday. $\quad(\mathrm{ReH})$

So everybody who replied was at a conference. ( $R a C$, Xena)

The scheme Xena (xea-1) may at first appear somewhat quirky, and yet, even though all valid syllogistic schemes are of equal value logically speaking (cf. below), it has a certain appeal. Besides Barbara, Xena is the only valid scheme of the first figure that ends with a universal $a$-conclusion, which one might deem attractive. Also, the scheme employs two self-converting (symmetric) premises, whence it is valid in all four figures, which is convenient.

Negation equivalent syllogistic schemes can be neatly represented in a cube of their own. The following syllogistic schemes of the first figure are equivalid.

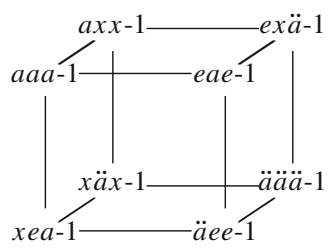

$$
\begin{gathered}
\text { with } \_ \text {indicating } P \text {-negation } \\
\quad \begin{array}{l}
\text { indicating } M \text {-negation } \\
\text { indicating } S \text {-negation }
\end{array}
\end{gathered}
$$


Thus, given the validity of one of the eight schemes, they are all valid.

The syllogistic schemes dealt with thus far all have general conclusions. Valid schemes with particular conclusions can be obtained by the principle of noncontradiction. If a conclusion follows from two premises then a negation of the conclusion entails that the premises cannot both be true. There are, of course, two options. We can take the negation of the major premise to follow from the negation of the conclusion and the sustained (but converted) minor premise, and also the negation of the minor can be seen to follow from the (converted) major and the negation of the conclusion.

\section{Observation 4 (Contraposition)}

- The syllogistic schemes $u v w-1, w^{\prime} v u^{\prime}-3$ and $w^{\prime} v^{c} u^{\prime}-1$ are equivalid (M-CP).

- The syllogistic schemes uvw-1, $u w^{\prime} v^{\prime}-2$ and $u^{c} w^{\prime} v^{\prime}-1$ are equivalid (m-CP).

Thus, and according to conventional wisdom, the validity of the classical Bocardo (oao-3) and Barocco (aoo-2) can be derived from that of Barbara by means of contraposition. Also Darii (ai -1 $_{\text {) }}$ and Ferio (eio-1) can be derived, from Celarent, that is.

\begin{tabular}{|c|c|c|c|c|c|c|}
\hline$M e P$ & & SiP & (M-CP) & SaM & (reord.) & $\operatorname{SaM}$ (conv. ) \\
\hline SaM & & SaM & & SiP & & PiS \\
\hline$\overline{\mathrm{SeP}}$ & (Celarent) & $\overline{M i P}$ & (Disamis) & $\overline{P i M}$ & (Datisi) & $\overline{P i M}$ (Darii) \\
\hline$M e P$ & & $M e P$ & $(\mathrm{~m}-\mathrm{CP})$ & PeM & (conv.) & \\
\hline SaM & & SiP & & SiP & & \\
\hline$\overline{S e P}$ & (Celarent) & $\overline{\text { SoM }}$ & (Festino) & $\overline{\text { SoM }}$ & (Ferio) & \\
\hline
\end{tabular}

Notice that if we have contraposition at our disposal, we do not need a separate arrangement for reordering premises. The premises of a syllogism can be permuted by contraposing the major of the syllogism first, then contraposing the minor of the result, and finally contraposing the major of that.

(11) The syllogistic schemes $u v w-1$ and $v^{c} u^{c} w^{c}-1$ are equivalid by M-m-M-CP. ${ }^{7}$

Observation 1, in other words, is a corollary of Observation 4.

If we confine ourselves again to syllogistic schemes of the first figure, the eight general syllogisms that we displayed in a cube above give us two times eight particular ones, by major and minor contraposition, respectively. These can be displayed in a cube as well.

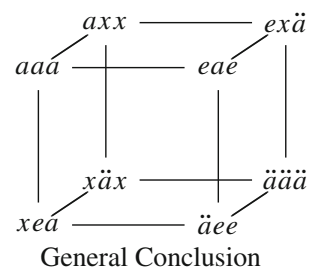

General Conclusion

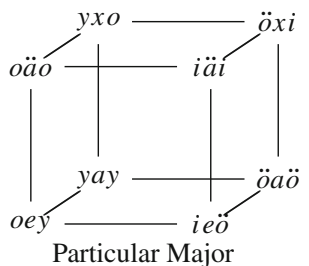

Particular Major

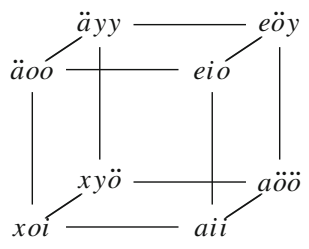

Particular Minor

\footnotetext{
${ }^{7}$ By performing major-minor-major contraposition on $u v w-1$ we obtain $v^{c \prime \prime} u^{\prime \prime c} w^{\prime c^{\prime}}-1$, which is $v^{c} u^{c} w^{c}$, as is easily checked; likewise, minor-major-minor contraposition, m-M-m-CP, gives $v^{\prime \prime c} u^{c \prime \prime} w^{c^{\prime}}-1$, which is $v^{c} u^{c} w^{c}$ as well.
} 
(The two new cubes are the CP-images of the original one. The legend explicating the lines therefore changes, but in a systematic fashion of course. For instance, in the cube in the middle the vertical lines indicate $S$-negation, and in the cube on the right they indicate $P$-negation.) We have by now derived 24 syllogistic schemes of the first figure, and, by conversion, four times that number in total. Actually, they are all the valid schemes. A more general observation may serve to substantiate this claim. ${ }^{8}$

Observation 5 (Polarity) A syllogistic scheme is valid iff

(1.) the middle term $M$ occurs both negatively and positively in the premises,

(2.) the polarities of the major term $P$ and the minor term $S$ in the conclusion align with those of the two terms in the premises, and

(3.) there is at most one particular premise, and then, and only then, also a particular conclusion. ${ }^{9}$

In the outline of the proof we have already employed the fact that the syllogistic schemes displayed in the three cubes, and their conversions into the other figures, are all valid schemes. It also shows they are the only valid ones.

The generalization stated here may serve to adjust a common and old conviction about syllogistic validity. According to received wisdom, first, a scheme can be valid only if it has at least one positive premise, and, second, a valid scheme with a negative premise can only yield a negative conclusion. Both generalizations are correct if restricted to syllogisms obtained from the square of opposition, but they do not hold in general, as we see from the syllogisms obtained from the cube. A valid scheme can after all employ two negative premises: $\ddot{a} \ddot{a} \ddot{a}-1$ (Bärbärä), and $\ddot{a} e e-1$ (Cälerent) (this was also observed by Reichenbach 1952). Moreover, we can draw a positive conclusion from a negative premise. An example is the valid scheme $x \ddot{a} x-1$ (Xäx), and we also have Xena (xea), again. Xena has an $a$-conclusion which is positive, or affirmative, without any doubt, and the minor $e$-premise is undisputably negative.

The present findings also lead up to the following conclusion. The validity of a syllogistic scheme in any figure can be traced back to that of one in any other figure. Moreover, the valid schemes of the first figure can be derived from only one. Thus:

Observation 6 (One for All) All valid syllogistic schemes from the cube can be derived from any one of them by means of conversion, negation and contraposition. ${ }^{10}$

\footnotetext{
${ }^{8}$ It is hard to believe that others have not made this generalization, so, in lack of having seen it stated thus before, I hereby attribute it to common sense.

9 Outline of the proof. If: By a mere inspection of the numbers of types of possibilities one can conclude that out of the $8 \times 8 \times 8 \times 4$ syllogistic schemes precisely $24 \times 4$ satisfy the conditions mentioned. (Condition 1 eliminates $1 / 2$, condition 2 eliminates $1 / 2$ twice, and $3 / 8$ of the possibilities satisfy condition 3 .) In the first figure these are seen to be the 24 schemes displayed in the three cubes above, and since these are equivalid, and at least one of them is valid, they are all valid. Their conversions to the other three figures preserve both the mentioned conditions and validity.

Only if: By contraposition. If one of the first two conditions is not satisfied, we can present a case (with $M$, $S$, or $P$ universal or empty) in which the conclusion is trivially false while the premises are contingent, or in which the premises are trivially true, and the conclusion is contingent. In either case the conclusion can be false while the premises are true. If both premises are particular no independent conclusion follows. If both premises are general, no particular conclusion follows.

10 Proof. The observations in this section are all stated as equivalences or equivalidities, whence they are convertible. Now suppose the validity of some scheme $u^{*} v^{*} w^{*}-i$ is not derivable by means of the three
} 
This is a conceptually welcome result about the syllogistics of the cube. Notice that a completely analogous result can be stated for the restricted set of validities emanating from the square. The slightly disturbing point is that the structural principles involved have only a limited application within the confines of the square.

By conceiving of the square of opposition as just a side of the cube of opposition we have been able to give a general characterization of the validity of a syllogistic scheme. The findings are not groundbreaking, and surely do not go beyond anything like elementary set-theory, but perhaps the phrasing of the results has been illuminating, or so we hope. Reichenbach (1952) had already shown that the validity of all cubistic syllogisms can be traced back to that of Barbara. We may now perceive more clearly that this means that it is not only Barbara that can do the designated job. Celarent, or Xena, might serve equally well, or even Remöny ( $e \ddot{o} i-1)$, if one enjoys the following inference.

Nobody on this list is accepted.

$(L e A)$

There were not only rejects on the list. $\quad(R \ddot{o} L)$

So, some are neither rejected nor accepted. (RyA, Remöny)

As I will argue in the next section, more is to be gained from the more general perspective adopted here. An arguably more semantic investigation of the cube, not of the square, forms the start of a fresh look upon quantified structures in natural language.

\section{Generalized Quantifiers}

In this section the combinators from the cube are taken up in the wider theory of generalized quantifiers. In the first Sect. 2.1 give a characterization of the combinators as determiners featuring characteristic semantic properties. The features characterizing the cube are next shown to be more widely relevant for the analysis of quantified constructions in natural language. It will be seen that some of the phenomena described cannot be properly described, or even perceived, if it is assumed that the combinators belong to the square, or that determiners more in general ought to be 'conservative'. The last subsection discusses somewhat more extensively this common, but upon reflection too limiting, assumption that natural language determiners actually are conservative.

\subsection{A Generalized Quantifier Characterization of the Cube}

The theory of generalized quantifiers is concerned with the study of quantifiers and determiners in natural and formal languages. The primary interest is in the semantic conception of a determiner, set-theoretically understood as a relation between sets of individuals.

Footnote 10 continued

structural observation. Then it can be turned into one of the form $u v w-1$ which is also not derivable, and which does not satisfy the three conditions stated in the previous observation. It is, hence, invalid, and $u^{*} v^{*} w^{*}-i$ is likewise. 
Definition 1 (Determiners) Given a universe of individuals $E$, a determiner $D_{E}$ on $E$ is a relation on $\mathcal{P}(E)$, i.e., $D_{E} \subseteq(\mathcal{P}(E) \times \mathcal{P}(E)) .{ }^{11}$

(I write $D_{E}(A, B)$ iff $\langle A, B\rangle \in D_{E}$.) Our primary interest is in the semantic conception of determiners, and their syntactic realization, as lexical determiners or otherwise, is of secondary interest. Even so, syntactically, a determiner can be taken to combine with two expressions, understood as denoting sets, so as to produce a sentence. The resulting sentence is true if and only if the determiner, semantically conceived, holds of the pair of associated sets. These sets are often, but not exclusively, denoted by a nominal and a verbal phrase, respectively.

The categorial combinators we have seen in the previous section are essentially determiners. The corresponding set-theoretical objects are called by distinguished names, the first six of them fairly standard. That is, we have NO (e), SOME $(i), A L L$ $(a), N O T \_A L L(o), O N L Y(\ddot{a})$, and NOT_ONLY $(\ddot{o})$; the less common ones are phrased here as $\operatorname{UNLESS}(x)$ and $E L S E(y)$. The theory of generalized quantifiers enables a straightforward and general characterization of the cube, as well as of its sides.

Determiners can be deemed 'logical' in a strictly defined sense. This property is stated in terms of the notion of a bijection or permutation $\pi$, to make its independence of individual and contingent properties clear. (In this definition, for any bijection $\pi$ from $E$ to $E^{\prime}$ and $A \subseteq E, \pi(A)=\{\pi(e) \mid e \in A\} \subseteq \pi(E)$.)

(13) A determiner $D$ is logical iff $D_{E}(A, B)=D_{\pi(E)}(\pi(A), \pi(B))$, for any bijection $\pi$ from $E$ to $\pi(E)$.

Logicality of a determiner effectively means that it is independent of any specific properties of the individuals in the universe. This becomes clear from the following observation. (van Benthem 1983)

(14) A determiner $D$ is logical iff $D_{E}(A, B)$ depends only on the cardinalities of $A \backslash B, A \cap B, B \backslash A$ and $E \backslash(A \cup B)$.

Even though the definition is fairly technical, the notion of a logical determiner itself is quite natural. A logical determiner $D$ only holds of two sets $A$ and $B$ in $E$ because of an independently definable set-theoretic relation between $A$ and $B$ in $E$. Modulo some idealization involved in their application to sets, this appears to be true of most determiners in natural language. Observe that the determiners associated with the combinators from the cube count as logical, since for any associated determiner $D, D_{E}(A, B)$ only depends on the mentioned cardinalities. E.g., $N O_{E}(A, B)$ holds iff $|A \cap B|=0, A L L_{E}(A, B)$ holds iff $|A \backslash B|=0$, and $E L S E_{E}(A, B)$ holds iff $|E \backslash(A \cup B)| \geq 1$, etc.

The eight categorial combinators can be characterized as logical determiners that are minimally sensitive to extension of the universe. If an element is added to a universe $E$ it is added to either one of the four subsets relevant for the valuation of a determiner. A determiner counts as minimally sensitive to addition if such an addition affects the valuation of the determiner only once.

\footnotetext{
11 It may, and will, happen, that the universe $E$ gets contingently, contextually, restricted to a subset $C \subseteq E$, so that $A$ and $B$ are no longer guaranteed to be a subset of the universe. In that case I write $D_{E \cap C}(A, B)$ iff $D_{E \cap C}(A \cap C, B \cap C)$.
} 
Observation 7 (Characterization of the Cube) The cube hosts the logical determiners that are minimally sensitive to extension of the universe.

The Venn-diagrammatic representations in Sect. 2 show that $D_{E}(A, B)$, when true, may get falsified when exactly one individual is added to one of the distinguished areas-OR, when false, may get verified by one and only one such an addition. These are exactly the eight possible casualties. I will call these eight determiners the minimal logical determiners in what follows.

The possible impact of an extension of the universe enables us to distinguish the combinators from the top and the bottom plane of the cube.

Observation 8 (Top and Bottom Plane) The top plane of the cube hosts the minimal logical determiners which can be falsified through an extension of the universe; the bottom plane of the cube hosts those that can be verified.

We can identify the four other sides of the cube by means of some other general, characteristic, semantic properties of determiners, as we will see presently.

The domains or universes of quantification employed in natural language are normally contextually determined, or constrained, and the first argument of a determiner can be taken to establish that domain. If a determiner $D$ is used that way I will refer to it as yielding the nominally restricted reading $D^{n}$ of $D$. (Such a reading is, slightly misleadingly, called 'nominally restricted', because in paradigmatic uses of determiners the first argument is supplied by a nominal phrase.) It is defined as follows.

Definition 2 (Nominal Restriction) The nominally restricted reading $D^{n}$ of a determiner $D$ is defined by

$$
\text { - } \left.D_{E}^{n}(A, B) \text { iff } D_{E \cap A}(A, B) \text { (that is, iff } D_{A}(A, A \cap B)\right) \text {. }
$$

A nominally restricted reading of a determiner makes us look at the individuals in the extension of the determiner's first argument only-as if $E=A$, and if the determiner is logical its interpretation only depends on the cardinalities of $A \backslash B$ and $A \cap B$.

If we look at the cube of oppositions, we find that the quantified propositions at its front are immune to nominal restriction, and those at the back are allergic to it. A determiner $D$ is here said to be immune for a certain operation if and only if the operation does not change its interpretation: $D=D_{n}$; the determiner is said to be allergic to it if and only if the operation trivializes it, i.e., if and only if it renders one or both of its arguments redundant.

Observation 9 (Front and Back Plane) The front plane of the cube hosts the minimal logical determiners that are immune to nominal restriction; the back plane host those that are allergic to it.

Thus, quite obviously, and as is well-known, the following pairs of sentences are basically equivalent

(15) Some boy danced. / Some boy is a boy who danced.

(16) Every man is rational. / Every man is a man who is rational.

Conversely, while (17) is contingent, (18) is trivially false. 
(17) Not only boys danced.

(18) Not only boys are boys who danced.

The explanation for the difference is that $S O M E$ and $A L L$ are immune to nominal restriction, and NOT_ONLY is sensitive to it, even allergic.

Of course, the domain of quantification can also be restricted, systematically, by the second argument of a determiner, thus yielding so-called verbally restricted readings of determiners. (These readings are, also slightly misleadingly, called 'verbally restricted', because in paradigmatic uses of determiners the second argument is supplied by a verbal phrase.)

Definition 3 (Verbal Restriction) The verbally restricted reading $D^{v}$ of a determiner $D$ is defined by:

- $D_{E}^{v}(A, B)$ iff $D_{E \cap B}(A, B)$ (that is, iff $\left.D_{B}(A \cap B, B)\right)$.

Verbal restriction makes one look at the individuals in the extension of the determiner's second argument only-as if $E=B$. If $D$ is a logical determiner, $D^{v}(A, B)$ only depends on the cardinalities of $A \cap B$ and $B \backslash A$. Verbal restriction serves to distinguish the left and right sides of the cube.

Observation 10 (Left and Right Plane) The left plane of the cube hosts the minimal logical determiners that are immune to verbal restriction; the right plane hosts those that are allergic to it.

The following pairs of examples are, while slightly odd perhaps, extensionally equivalent.

(19) No boys were dancing. / No dancing boys were dancing.

(20) Only girls are rational. / Only rational girls are rational.

Conversely, while (21) is contingent, (22) is trivially true.

(21) All boys were dancing.

(22) All dancing boys were dancing.

The explanation for the difference is analogous to the one above. ${ }^{12}$ It turns out, then, that the six sides of the cube each accommodate determiners with distinctive semantic properties. These properties, in turn, correlate systematically with particular linguistic properties of natural language determiners, as I will argue in the next subsections.

\subsection{Linguistic Applications}

Consider a situation in which an exam is at issue, and the question is how the students did.

(23) What about the exam? How did the students do?

\footnotetext{
12 Surely the restricted rephrasings in the examples here-rephrasings which are rendered equivalentcarry additional meaningful overtones, due to induced information structure and assumed intonation. I will come back to this in the next section.
} 
Here is a list of some possible replies which are surely felicitous.

(24) Some failed. / None failed. / All failed.

Everyone failed. / Not all failed. / At most five failed.

Many failed. / Not many failed. / Most failed.

It almost seems like any intended reply of the form "Det $A B$ " figures well when its nominal argument $A$ is deleted. ${ }^{13}$ However, the following replies are out, if not indeed plainly ungrammatical.

(25) ? Only failed. / ? Not only failed.

This is surprising, because a full reply appears to be fine.

(26) Only students failed.

Not only students failed.

If we, for convenience and for the time being, equate the syntactic expression of a determiner with the determiner itself, we can come to the following observation.

Observation 11 (*Noun-Deletion) Determiners allergic to nominal restriction resist deletion of their nominal argument.

I conjecture that if a determiner is allergic to nominal restriction, its nominal argument constitutes its focal contribution, whence the argument cannot be sensibly deleted. It is not easy to test this generalization, because it is hard to find natural language determiners, besides "only" and "not only", that are allergic to nominal restriction. However, "mostly" appears to be one. MOSTLY can be defined as the converse of $M O S T$, so that $\operatorname{MOSTLY}_{E}(A, B)$ if and only if $\operatorname{MOST}_{E}(B, A)$ if and only if most of the $B$ 's are $A$ 's. Upon this interpretation, MOSTLY is allergic to nominal restriction, and indeed we find that "mostly" resists deletion of its nominal argument.

(27) ? Mostly failed.

Notice that, again, there is no problem with the use of "mostly" in a full reply.

(28) Mostly students failed.

The intuitions about the examples (24-28) seems to be fairly solid, and it may come as a surprise that the observation has, for as far as I am aware of, not been detected before. One of the reasons for this surely is that so few natural language determiners are allergic to nominal restriction. However, besides that, there is a strong theoretical inclination only to look at determiners that are immune to nominal restrictionto conservative determiners, that is-, thereby rendering the observation principally unobservable.

Considerable attention in linguistics has been devoted to the type of (quantified) noun phrases that may go into so-called "existential-there" constructions, and, for some people accordingly, into "relational-have" constructions as well. There are rather pertinent judgments that the sentences in (29) are all fine, and that those in (30) are ungrammatical, or marked, or at least require a somehow shifted interpretation.

$\overline{13}$ With the proviso perhaps that "The _ failed" be, obligatorily, rendered as "They failed." 
(29) There are some / no / five / many students.

Arnold has some / no / five / many sisters.

(30) There are ? all / ? not all / ? the $/$ ? most students.

Arnold has ? all / 'not all / ' the / ? most sisters.

There are quite a few speculations about the contrasting judgments. A semantic explanation has been pursued in the theory of generalized quantifiers, which has set out to identify characteristic semantic features of the determiners that are licensed in these constructions. There is some consensus that these constructions are felicitous if (and only if) the embedded determiners are intersective (and hence symmetric), as proposed by, e.g., Keenan 1987. This observation appears correct, but, as (Keenan 2003) notes, as long as one only takes determiners into account that are immune to nominal restriction. (Or 'conservative', cf. below.) However, "only" is not conservative, nor symmetric, but it figures well in the constructions at issue, as (Johnsen 1987) observed. The same goes for "not only".

(31) There are only / not only students.

Arnold has only / not only sisters.

A more general characterization of the licensed determiners appears to be called for. (Keenan 2003) proposes conservativity in the second argument as a characteristic feature, a property of determiners we label that of being 'regressive' below, and which very closely corresponds to immunity to verbal restriction. This may be too strong a requirement, however, because the necessary condition does not seem to be immunity to verbal restriction, but rather the weaker condition of not being allergic to it.

The existential contexts we are considering here in a sense annul, or trivialize, the second argument of the embedded determiners. If this is correct it is to be expected that determiners allergic to verbal restriction do not figure well there, whence the following tentative generalization.

Observation 12 ( ${ }^{*}$ E-there and ${ }^{*} R$-have) Determiners allergic to verbal restriction resist existential-there and relational-have configurations.

Looking at the minimal logical determiners from the cube, those on the left side are immune to verbal restriction, and figure well in the types of construction at issue. As hypothesized, the same goes for the non-minimal determiners that are not allergic to verbal restriction. Thus, "(at least / at most) five", and "many" behave as hypothesized and also "mostly" turns out to be fine in these contexts.

(32) There are many / mostly students.

Arnold has many / mostly sisters.

Interestingly, "many" is not unequivocally immune to either nominal nor verbal restriction, but it is surely not allergic to either one of them. (See the discussion in the next section.) The generalization, thus, appears to be correct. (Let me emphasize that it is not the purpose of the present paper to supply a compositional semantic analysis of existential-there constructions, or of that of relational-have constructions. I here confine myself to stating the relevant observations. ${ }^{14}$ )

14 There appear to be conflicting intuitions about a determiner-like compound "none of the", which combined with a noun phrase and a verb phrase makes up a sentence, like "None of the cats meowed." 
It appears that not even Keenan's generalization has caught on in the literature, and this may arguably be because of the persistent intuition that natural language determiners are conservative. (Cf., discussion in Peters and Westerståhl 2006; the issue is taken up in Sect. 3.5.) For upon this assumption the initial generalization is provably correct. If a determiner is (as is generally assumed) conservative, and if it has to be regressive (as Keenan requires for the configurations at issue), then the quantifier must be intersective and hence symmetric, as is easily proved. Thus we find that symmetry shows up again as a characteristic feature allowing determiners to figure in existentialthere constructions in, e.g., (Peters and Westerståhl 2006; Westerståhl 2014). However, note that the last generalization is tenable only upon a theoretical assumption-about conservativity of natural language determiners - and that it renders potentially relevant facts out of scope.

The felicity of quantified noun phrases in existential-there and relational-have constructions may appear to be some (marginal, Germanic) idiosyncracy, but the observations can be generalized. We seem to find a similar pattern in contexts induced by transitive intensional verbs, e.g., verbs of creation or intention. Consider the following examples.

(33) Muriel painted some / no / only / not only / five / many apples.

(34) Menno imagined some / no / only / not only / five / many unicorns.

(35) Mandy wants some / no / only / not only / five / many cars.

(36) Marc worships some / no / only / not only / five / many Goddesses

The object noun phrases in these examples have distinguished non-extensional readings. The noun phrases then do not relate to, or quantify over, a domain of actual apples, unicorns, horses or Goddesses. The verbs as it were create the domainsdomains, so to speak, in which there are no, only, not only, five or many apples, unicorns, etc. to be found. A similar construal appears inconceivable with noun phrases headed by "the", "all" and "most".

(37) Muriel painted the / all / most apples.

(38) Mandy wants the / all / most apples.

These last two types of examples can only relate to a domain of apples such that Muriel painted (all or most of) them, or such that Mandy wanted (all or most of) them. The apples at issue can very well be imaginary, or painted themselves, but they must figure in a given domain of things, a number of which is being ascribed the property

\footnotetext{
Footnote 14 continued

Interestingly, precisely the discord can be explained by the present generalization. For observe that there are two ways of understanding the embedded determiner "the", yielding two different analyses of the compound. The definite article can be taken anaphorically, yielding a presupposition of there being cats, in the universe or under discussion. A verbally restricted reading of the determiner in "None of the cats meowed." could thus be taken to express the proposition that, among those given cats none in the domain of meowers meowed, i.e., that none of them meowed. This is a non-trivial proposition, the compound thus is not allergic to verbal restriction, and hence "There are none of the cats." is rendered felicitous upon the anaphoric reading of "the cats." Alternatively, the embedded article can be read as yielding a quantificational reading, to the effect that "None of the cats meowed" yields the conjunctive proposition that there are cats and that none of them meowed. The verbally restricted reading then reads "There are cats who meowed, and none of them meowed" which is inconsistent. Attempting this reading, the construction would be infelicitous in an existential-there context. For as far as both readings are possible, both judgments can thus be argued for.
} 
of being painted, or wanted, etc. Not so for the previous examples, upon the reading envisaged.

It is not the purpose of this paper to supply an analysis of these constructions. (But, cf., e.g., Moltmann 2013, Ch. 5.) They are mentioned here only to point out that the very same semantic property serves to identify the noun phrases that do, or that do not, allow for such a non-extensional interpretation. The ones that do not are precisely the noun phrases headed by a determiner allergic to verbal restriction. Notice that, again, the proposed distinctive feature would be left unidentified if the determiners involved are assumed to be conservative.

\subsection{Many Readings}

The observations in the preceding subsection can be ignored by simply denying the existence of non-conservative determiners. "Only" is not a determiner falsifying the symmetry generalization about existential-there configurations simply because it is not a determiner, or so it can be said. Things are not that simple when we turn to the English lexical item "many". The item "many" can combine with a nominal and a verbal predicate so as to yield a sentence, like other determiners in natural language do. Semantically it can also be taken to behave like a determiner, i.e., as relating two sets. It is famously focus sensitive, but it is also topic sensitive, and it is not obviously immune to either nominal or verbal restriction. This fact makes it interesting for the present discussion.

Without further appeal to other resources, a sentence "Many $A B$ " can be taken to state in a model $E$ that the number of $A$ 's who are $B$ is more than some average in $E$. The following characterization is just as unobjectionable as unsatisfactory. (Just as much as an initial characterization should be.)

$$
\operatorname{MANY}_{E}(A, B) \text { iff }|A \cap B|>A V R_{E} \cdot{ }^{15}
$$

This analysis may appear unsatisfactory, because of this oracular $A V R_{E}$. Interestingly, the average can be seen to be structurally regimented by linguistic means. As has been observed at many places in the literature, MANY allows for a variety of proportional readings which can be elicited through focal emphasis, or topical deemphasis. People's intuitions seem to converge on the observation that a sentence "Many Belgians are cycling." can be used to state at least three things, roughly as follows.

(40) Many Belgians are cycling.

There is big number of Belgian cyclists.

(41) Many Belgians are cycling.

There are many cyclists among the Belgians.

(42) Many Belgians are cycling.

There are many Belgians among the cyclists.

\footnotetext{
15 Barwise and Cooper (1981) have proposed roughly one-third of the cardinality of $E$ as an initial, default, standard of $A V R_{E}$ - a number that of course is supposed to be variable.
} 
It is striking indeed that these are precisely the three types of readings which one may get from the initial characterization of $M A N Y$, by means of nominal and verbal restriction, respectively. For observe, if $B, C \subseteq E$ :

\section{Observation 13 (Proportionally Many)}

(43) $M A N Y_{E}^{n}(B, C)$ iff $|B \cap C|>A V R_{B}$;

(44) $\operatorname{MANY}_{E}^{v}(B, C)$ iff $|B \cap C|>A V R_{C}$.

Upon a nominally restricted interpretation, associated with (41), the number of Belgian cyclists is compared to an average among the Belgians; upon a verbally restricted interpretation, associated with (42), the number of Belgian cyclists is compared to an average among the cyclists. The truth-conditions are obviously, and intuitively, quite different.

A nagging question of course is what this ' $A V R_{X}$ ' eventually is, or how it should be determined. A substantial part of a reply to this question in specific circumstances of use will surely draw from highly contextual features such as (ir-)relevance, attention, expectation, and so on. But it seems to be generally agreed upon that focal emphasis on linguistic items in such constructions induces alternatives for the focused phrases, which can be used to set a standard for evaluation (cf., e.g., Cohen 2001; Greer 2014).

For instance, example (41) induces, or implicates, or relates to, a set of contextually alternative things that the Belgians might be doing, such as running, skiing, climbing, and what have you. Likewise, example (42) can be taken to relate to cyclists of alternative nationalities, such as the Dutch, Frenchmen, Germans, etc. Such alternatives can be used to provide a specific standard to literally count as the average. For example (41), with interpretation (43), we can define $A V R_{B}$ as follows.

$$
\begin{aligned}
& A V R_{B}=A V R_{D \in A L T(C)}|B \cap D|, \text { with } \\
& -A L T(C)=\{C Y C L E, R U N, S K I, C L I M B, \ldots\} .
\end{aligned}
$$

With 14 Belgian cyclers, 5 Belgian runners, 7 Belgian skiers, and 18 Belgian climbers, the average is 11 and the sentence would turn out true. Analogously, for example (42), with interpretation (44), $A V R_{C}$ can be defined as follows.

(46) $A V R_{C}=A V R_{D \in A L T(B)}|D \cap C|$, with

- ALT $(B)=\{$ BELGIAN, DUTCH, FRENCH, GERMAN, . . $\}$.

This time it is the average number of cyclists of other than the Belgian nationality that are relevant for the truth of (42). Both analyses may appear to be overly calculating, but on the right intuitive track.

It may hardly need comment that the determination of the average or standard in actual cases of use may depend on more than just the extensions of the terms employed here, and it has been argued that, of course, subjective, intensional features, expectations, play a role as well. (Fernando and Kamp 1996; Bastiaanse 2014) There can be overt disagreement on the choice of alternatives that one on occasion takes into account, which is not a (syntactically) given. However, it is important to note that all such qualifications, also depend on the particular choice for either a nominally or a verbally restricted interpretation of "many", a choice which, hence, cannot be neglected in any analysis. 
There is a considerable literature on the generalized quantifier interpretation of "many", that aims to show that it is essentially intensional, and trying to rescue the idea that it is conservative (cf., the recent Bastiaanse 2014; Greer 2014 for discussion). The present discussion may serve to show that many if not most of the specific findings in the literature can actually be obtained from a very rude and simple, plainly extensional, interpretation, as in (39). The readings discussed here are derived from principles, assumed generally available, of nominal and verbal restriction. Notice that these results would not obtain if it were assumed from the very start that "many" is conservative, that is, immune to nominal restriction.

\subsection{Only a Determiner}

It must be beyond doubt that the lexical item "only" can be used in combination with a common noun phrase $N$ and a verb phrase $V$, to yield something like a sentence, one that can be used to assert that, indeed, only $N$ are $V$, i.e., that all those who $V$ are $N$. "Only students sleep." simply says that all sleepers are students. As we have seen above, such an interpretation of "only" as ONLY (or $\ddot{o}$ ) may serve to explain why, on the one hand, it does not allow its nominal argument to be deleted, or elided, and also why, on the other, it does figure well in existential-there contexts. ${ }^{16}$

It has been observed that, while "only" is immune to verbal restriction, it is focus sensitive. As we have seen with the determiner "many", non-focal material can be assumed to be backgrounded, in that the material is projected into the domain of quantification. Even though we have also seen that "only" is allergic to nominal restriction, we do find partial instances of nominal restriction. Partial restriction can be defined, for all determiners, along the following lines. In the following definition I use $\langle A\rangle B$ to indicate a structured nominal argument with a backgrounded condition $A$, and focal material $B$.

Definition 4 (Backgrounding) The backgrounded reading $D^{b}$ of a determiner $D$ is defined by:

- $D_{E}^{b}(\langle A\rangle B, C)$ iff $D_{E \cap A}(B, C)$ (that is, iff $\left.D_{E \cap A}(B \cap A, C \cap A)\right)$.

Backgrounded (normally de-emphasized) material $A$ is 'projected' (as some would say) into the domain of quantification, leaving the material that is also $B$ as the first argument of the determiner. We may subtly, but significantly, observe that this kind of projection, or backgrounding, can be assumed to be generally available in all compound constructions, but without this having any effect if the construction hosts a determiner immune to nominal restriction. (This is easily seen.) However, in construction with a determiner which, like "only", is not immune to nominal restriction, this backgrounding has a substantial and interesting effect.

\section{Observation 14 (Backgrounded Only)}

\footnotetext{
16 Thus, the observation "There are only students.", can be rendered by "Everybody is a student." and the observation "There is only fish (on the menu)." amounts to the observation that "There is nothing else but fish." Let me emphasize again that it is not the purpose of the present paper to supply a compositional semantic analysis of existential-there constructions. It suffices to observe the relevant equivalences.
} 
- $O N L Y_{E}^{b}(\langle A\rangle B, C)$ iff $B \supseteq(C \cap A)$.

Consider a simple example.

(47) Only Belgian cyclists passed the test. $\left(O N L Y_{E}(B C, P)\right)$

Without any further restrictions, this says that if someone passed the test it was a Belgian cyclist, i.e., that all those who passed the test were Belgian. $((B \cap C) \supseteq P)$

(48) Only Belgian cyclists passed the test. $\left(O N L Y_{E}^{b}(\langle C\rangle B, P)\right)$

With emphasis on "Belgian", or by de-emphasizing "cyclists", we obtain the reading that if a cyclist passed the test it was a Belgian, i.e., that all cyclists who passed the test were Belgian. $(B \supseteq(P \cap C))$

(49) Only Belgian cyclists passed the test. $\left(O N L Y_{E}^{b}(\langle B\rangle C, P)\right)$

With emphasis on "cyclists", or by de-emphasizing "Belgian", we obtain the reading that if a Belgian passed the test it was a cyclist, i.e., that all Belgians who passed the test were cyclists. $(C \supseteq(P \cap B))$

"Only" is also notorious for applying cross-categorially, and what appears to be an independent research tradition is devoted to the study of the so-called 'focus-sensitive' uses of "only". ${ }^{17}$ In this tradition, which hosts a whole variety of sophisticated analyses—syntactic, semantic, pragmatic or otherwise-, it is commonly assumed that the focus-sensitive uses of "only" amount to universal quantification over alternative propositions obtained from alternatives of the focused material. "Only" is conceived of as applying to structures (ideally sentential) containing a focused element $B$, as in $\left[s \ldots[B]_{F}---\right]$, and its interpretation, in effect, can be characterized, schematically, as follows. ${ }^{18}$

(50) $O N L Y\left[S \ldots[B]_{F}---\right]$ iff if $\left[s \ldots[X]_{F}---\right]$ then $X=B$

for all available alternatives $X$ of $B$.

The type of sentences that fit this scheme induce sets of alternatives to the focally emphasized phrase, to the effect that only the focused phrase itself, and not any of its alternatives, constitutes a verifying instance of the structure. Obviously, any actual interpretation of such 'exclusive' sentences needs a specification of the domain of alternatives that these sentences are assumed to be quantifying over, probably analogous to the ones employed in the focus-sensitive construal of "many" above.

It can be observed that this way of rendering the interpretation of focus-sensitive "only" is very close to its generalized quantifier interpretation, if not essentially the same. Let us consider a simple example.

(51) Harry was only swimming.

According to the sketched schematic analysis the sentence can be used to claim that if there is anything that Harry did, it is swimming. This, however, is formally and intuitively equivalent with any one of the following two paraphrases.

\footnotetext{
17 This tradition finds its roots in the seminal (Rooth 1992; Krifka 1993) and also the thorough and often cited (van Rooij and Schulz 2007). The authors, as it happens, do not discuss any use of "only" in a determiner-like role.

18 I only give a schematic reconstruction of the crucial parts of the schemes; a compositional analysis of the sentences actually hosting "only" is much more involved of course.
} 
(52) All that Harry did was swimming.

(53) (Harry was swimming.) And (he did) nothing else.

Strikingly, if (51) comes out true under some determination of the domain of alternatives, then (52) will come out true as well, under the very same choice of alternatives. It is surprising, perhaps, how neatly such analyses fit the generalized quantifier interpretation of "only". For if the focalized phrase in the scope of "only"$S$, for "swimming" in the example above-is not used as a predicative noun, but taken as a definite focal object, we can take the property $\lambda X X=S$, the property of being that object, as the focal meaning. Then the domain of quantification $D=A L T(S)$ can be taken to be that of the contextually relevant alternatives to swimming. Thus, in line with a focus-sensitive-account of "only", the truth-conditions of (51) can be adequately rendered by the generalized quantifier specification.

(54) $O N L Y_{A L T(S)}(\lambda X X=S, \lambda X X(h))$.

Clearly this is equivalent with requiring that all that Harry did, among all alternatives conceived, was swimming, as stated by (52).

(55) $A L L_{A L T(S)}(\lambda X X(h), \lambda X X=S)$.

Moreover, we can assume that the arguments of the determiners in (54) and (55) are not vacuous, which is a truly Aristotelian assumption. This would imply that there is a property among the alternatives in the domain of quantification and such that Harry has or had it. Thus, both (54) and (55) imply that that is the property of swimming, to the effect that the presupposition is that Harry was swimming. This, as a matter of fact, is what most authors would like to see included among the truth-conditions of (51).

Analogously, example (56), rendered as (57), is equivalent with (58).

(56) Only Harry was swimming.

(57) $O N L Y_{A L T(h)}(\lambda x x=h, \lambda x S(x))\left(=A L L_{A L T(h)}(\lambda x S(x), \lambda x x=h)\right)$.

(58) If somebody was swimming, it was Harry.

There is some debate in the literature on the question whether example (56) asserts, or presupposes that Harry himself was swimming. Upon the present analysis in (57), a purely Aritotelian assumption of existential import would indeed induce the existential presupposition that somebody was swimming. In conjunction with what (57) asserts, viz. (58), this entails that Harry was swimming, as indeed required.

It may have to be emphasized once more, that, for any use of such exclusive sentences, most of the work goes into establishing the domain of quantification, that is the set of focal alternatives. In this paper I have little to say about that issue, and refer to the vast literature on the subject, e.g. (Rooth 1992; Krifka 1993; van Rooij and Schulz 2007; Cohen 2001; Greer 2014). I would like to point out, however, that the present approach applies fully generally. A whole variety of examples can be handled adequately in a way analogous to the one sketched above.

(59) There were only associate professors.

(60) Alfred only introduced Bill to Carol.

(61) You were only asked to check the knifes on the table.

(62) Only if a student works hard will he pass the exam. 
Example (59) can be handled as a backgrounding structure, like the one we find in (47). Example (60) is classical (Rooth 1992), and turns out to mean that if Alfred introduced anybody to Carol, it was Bill. (More formally: in the domain of relevant persons, only Bill is in the set of those who are introduced by Alfred to Carol, if any are.) Example (61) can be used to exclude from inspection knifes under, in, or behind, the table, if these are the contextually relevant options. Example (62) is an "only if donkey sentence" to be subjected to a dynamic semantic or discourse representation theoretic treatment. In (Dekker 2001) I have shown how this example can be analyzed and then yield the proposition that if there is anything a student can do to pass the exam, it is working hard (even though it is not a guarantee for success). Also this analysis shows up essentially along the lines sketched here.

We see that truly interesting semantic features of "only" can be stated in terms of the generalized quantifier $O N L Y$, that is, in terms of its Aristotelian interpretation $\ddot{a}$. The reader may wonder why all this has not been established before. The answer seems to be that research on focus-sensitive "only" has left the nominal uses of "only" out of its scope, and, with the positive exception of (de Mey 1991), the generalized quantifier approach has exclusively focused on determiners that are 'conservative', and "only" is obviously not that.

\subsection{Determiners, Conservative and Regressive}

The preceding findings-viz., on allergy to verbal restriction, a proportional reading of "many" and restricted readings of "only" - still don't make much sense if one maintains the assumption that all natural language determiners are conservative. As I have already indicated non-conservative items like "only" are often denied the status of a determiner in the literature. The phenomena observed could thus be taken to not even exist. In this Sect. 2.1 want to argue that the support for such claims is in no way conclusive, if not simply begging the question.

It is useful to first clarify the terminology employed. A determiner $D$ is said to be conservative iff always $D_{E}(A, B)$ iff $D_{E}(A, A \cap B)$, and it is said to be regressive iff always $D_{E}(A, B)$ iff $D_{E}(A \cap B, B)$. Now for any determiner $D$ that satisfies extension $\left(D_{E}(A, B)\right.$ iff $D_{E \cap(A \cup B)}(A, B)$, cf., below), we find that $D$ is conservative iff $D$ is immune to nominal restriction, and $D$ is regressive iff $D$ is immune to verbal restriction.

An initially appealing, but upon reflection hardly convincing, argument for taking conservativity as a universal property of natural language determiners, is that it serves to severely restrict the enormously large class of possible determiner denotations. On a universe of $n$ individuals the limitation to conservative determiners reduces the number of $2^{4^{n}}$ possible determiners to $2^{3^{n}}$, which is a substantial reduction indeed, and which has even been appealed to to account for the fact that we can actually learn the right denotations of determiner expressions in the acquisition of natural language. This argument loses much of its original appeal, though, once we realize that a child, in a universe with 3 individuals only (its mother, its father, and itself, for instance), is supposed to find himself faced with almost 135 million possible conservative determiner denotations. If we assume, perhaps more realistically, that a 
child would be designed to learn, for instance, minimal logical determiners, then the limitation to conservative determiners would reduce the number of possibilities from 8 to 4 . Useful, of course, but not significant. ${ }^{19}$

The expression "only", like the expression "many", has also been denied the status of a determiner, because, unlike other determiners, it is also used as an adverb, so it is an adverb, not a determiner, or so it is suggested. This is a curious argument, because, by the same reasoning, we may say that while "only" behaves like an adverb, yet, since it is also used as a determiner, it is a determiner, and hence not an adverb. Also, by the same type of reasoning we might say that "and", as in "Harry opened the door and Milly came in", is not a sentential connective because "and" can be used to conjoin expressions other than sentences. Independent motivation for such a claim thus should be provided, and the fact that the terms "only" and "many" can be used and interpreted like other determiners is prima facie evidence against it. ${ }^{20}$

There is no reason to doubt that most, if not almost all, lexically realized determiners are conservative, and that conservative determiners are more 'natural', and more 'easy to learn'. While this does not supply any evidence for conservativity as a universal semantic property of (lexical) determiners, it may help explain why many researchers are eager to suppose so. Van Benthem (1991, p. 30) observes "This universal already illustrates the earlier-mentioned interaction between semantic observation and semantic theory. (...) nowadays one finds that this failure of conservativity rather becomes one in an already existing list of reasons for not counting "only" as a determiner (but, rather, say, an adverb). Thus, successful pieces of theory exert pressure on previous empirical schemes of classification." We can indeed witness such interactions at work in syntax and semantics, but the reliance on conservativity is not, I think, as safe as it seems. A semantic universal will always be a welcome substantial discovery, but a theoretical convenience may turn into an inconvenience or even handicap if not carefully handled.

The interaction alluded to by van Benthem can be observed, for instance, in work on dynamic semantics and discourse representation that deals, among others, with 'donkey phenomena' in quantified and conditional constructions. Chierchia (1992, p. 151) argues that "weak readings [of quantified donkey-sentences, PD] are linked to conservativity and since the latter is universal, the former must be universal too." Even more outspoken are van Eijck and Kamp (1997, p. 225): "It is one of the central claims of DRT that this kind of anaphoric connection [in quantified donkey-sentences, $\mathrm{PD}$ ] is possible because the material of the quantifying sentences that makes up the

\footnotetext{
19 (Hunter and Lidz 2013) recently tested the relative learnability of two new lexical determiners with conservative meaning NOT_ALL and non-conservative meaning NOT_ONLY, respectively. The results indicated "a (marginally) significant dependency between conservativity of the determiner and success in learning" (p. 326). Interestingly, despite the authors' conviction that "[a] striking cross-linguistic generalization about the semantics of determiners is that they never express non-conservative relations" (p. 315), they had it that " 'only' refers to the interpretation (...) that reverses the inclusion expressed by 'all' (i.e. 'only girls are on the beach')." (p. 327). "Only" was said to be "not representable by a conservative determiner," (p. 327), though, apparently not because it is not conservative, but because 'only' is not a determiner (p. 332).

20 Even Peters and Westerståhl apparently agree that "only" has a determiner use, acknowledging that it is "an expression with much wider and more varied use." (Peters and Westerståhl 2006, p. 219, emphasismine)
} 
restrictor is also, implicitly, part of the quantifier's body. This principle also explains why natural language quantifiers are always conservative (...)."

Such claims, however appealing, as a matter of fact render the theories for which they are stated vulnerable. For if a proposed theory or analysis entails (predicts, explains, ...) conservativity as a universal, then the eventual detection of nonconservativity would actually falsify the theory or analysis. This worry is not just imaginary. Next to the so-called donkey phenomena in constructions headed by (universal) quantifiers and in if-conditionals, we witness analogous phenomena in constructions headed by "only" and in only_if-conditionals. (von Fintel 1994; Dekker 2001) The first are successfully dealt with in the mentioned theories, but by the claimed inalienable bond with conservativity the treatments are inapplicable to the second, by principle.

Fortunately, the situation is not that bad, because a successful, and uniform, dynamic or discourse representation theoretic treatment of both types of phenomena is feasible. (Dekker 2001) However, it appears that such can only be achieved once the claimed link between anaphora relationships and conservativity is given up. Thus, while there may be a theoretical urge or inkling to embrace a (quasi-)empirical generalization like that of the conservativity of natural language determiners, such urges, if not resisted, better be handled with caution.

Conservativity is a generally shared assumption that is often taken for granted. But it is mostly only taken for granted and it is almost never sanctioned, or actually or empirically verified. On the contrary, it seems, since leading advocates of conservativity themselves question its status as a semantic universal. Quite a few authors (von Fintel and Matthewson 2008; Francez and Ben-Avi 2014; Greer 2014; Herburger 1997; Keenan 1996; de Mey 1996; Zuber 2004) come to the conclusion that if determiners are not conservative, in their first argument it is said, they are assumed to be 'conservative in their second argument', that is, they are what I call 'regressive'. ${ }^{21}$ Obviously, if determiners are generally assumed to be 'conservative in their first or second argument', they are no longer required to be conservative in the original sense.

The relevant observations may lead to the formulation of a 'conservativity'constraint, or universal, that we can very well live with, but notice that it involves a terminological move that ought to be handled with care. If the term 'conservative' comes to mean what I take 'conservative or regressive' to mean, all previous claims about conservativity will have to be qualified or trashed. For, for instance, the statement that the quantifiers from the square are precisely those minimal logical determiners that satisfy conservativity would no longer be true. For, likewise, the claim that "only" is not conservative would no longer be true. And a claim to the effect that conservative determiners are easier to learn than non-conservative ones, would suddenly become an entirely different claim.

I prefer to stick to the use of the term 'conservative' in the way it was originally intended. But even so, independent of my preferences, the argument that "only" is not a determiner because it is not conservative will have to be qualified. Either the argument fails, because natural language determiners are no longer required to be conservative

\footnotetext{
21 Yet other qualifications of conservativity are also provided, see, e.g., (Kanazawa 1994; Bastiaanse 2014), but these nominal moves, too, may serve to emphasize that determiners are not generally conservative in the sense the discussion started out to be on.
} 
in the original sense. Or the argument fails because the premise is no longer true, and this is because "only" is deemed conservative after all, in its second argument, that is.

\section{Natural Se(le)ctions}

In the first section we have left the two-dimensional square behind us and studied the syllogic of the three-dimensional cube of oppositions. But it is not for no good reason, of course, that the square has ruled the logico-linguistic landscape. The four corners of the square make up a very natural selection from the cube, as has been observed in various linguistic domains. However, recent interest in Aristotelian 'hexagons' and 'dodecahedrons' may be indicative of the fact that the square might be too much of a straightjacket for the classification of natural language combinators. In this Sect. 2 briefly indicate some alternative subsections of the cube that can be distinguished. ${ }^{22}$

The Square of Opposition The four corners of the square show up distinctly in various guises. Germanic languages host some elementary quartets that are naturally accommodated in the square. In English we find "nothing", "everything", "something" and "not everything", and, similarly, "nobody", "everybody", "somebody" and "not everybody", and, likewise, "never", "always", "sometimes" and "not always". 23 It has been observed that, typically, English does not contain noun phrases like "onlything", or "onlybody", or an adverb "onlytimes", neither do related natural languages. The reason, however, is not, as seems to be appealing to assume, because ONLY and NOT_ONLY are not determiners, which we deem a far-fetched and unwarranted conclusion, but simply that they are determiners with the specific semantic characteristic property of being allergic to nominal restriction. As we have seen in Sect. 3.2, they resist emptying their focal first argument. Likewise for the temporal counterpart of "only". Trivially, we can only characterize these items as determiners with a distinctive semantic property, if we characterize them as determiners in the first place.

A Prism of Oppositions In the previous section we witnessed some attempts to characterize 'natural' or 'naturally occurring' logical combinators or determiners, in order to restrict logical space and to settle upon a 'tractable' portion of it. (Keenan 1996, fn. 1) seems to have been the first to argue that if determiners are not 'conservative', they are what we call 'regressive' and (Greer 2014) aptly implemented this idea in an arguably syntactic manner. We can cash out the idea semantically using a notion of extensional restriction. On the extensionally restricted reading of a determiner it is

\footnotetext{
22 I thus remain constrained myself, by the cube, and do not consider, e.g., Boolean meets or joins of its corners. Notice, however, that any Boolean meet (conjunction) of two general corners would violate differential import. "All and only", like "if and only if", is used to state an identity, which is trivial if not conceived of intensionally; likewise, a construction with "All and no" implies that the first argument argument is empty, and that the second, hence, is vacuous.

23 Here and above, I deliberately include the apparently compound expressions "not everything", "not everybody" and "not always". The reason is that the constituent "not" does not itself seem to figure as a productive lexical item or morpheme in these constructions. We can of course understand, for instance, "not nobody" and "not someone" and "not sometimes," but not as smoothly as we do understand the 'quasi-compounds' just listed.
} 
assumed that its first and second argument jointly constitute the universe, i.e., as if $E=A \cup B$.

Definition 5 (Extension) The extensionally restricted reading $D^{e}$ of a determiner $D$ is defined by:

- $D_{E}^{e}(A, B)$ iff $D_{E \cap(A \cup B)}(A, B)$.

We can observe that the minimal logical determiners UNLESS and ELSE are allergic to extensional restriction, and that the other six are immune to it.

Observation 15 (Extension) A minimal logical determiner $D$ is immune to extensional restriction iff $D$ is NO, ALL, ONLY, SOME, NOT_ALL, or NOT_ONLY (i.e., $e, a, \ddot{a}, i, o, \ddot{o})$.

Immunity to extensional restriction implies that if $A, B \subseteq E \subseteq E^{\prime}$, then $D_{E}(A, B)$ if and only if $D_{E^{\prime}}(A, B)$. (Actually this is the standard formulation of the condition for a determiner to 'satisfy Extension', whence the name.) The six mentioned logical determiners that are immune to extensional restriction hold of two sets $A$ and $B$ independent of the wider universe 'surrounding' these two sets.

It has been claimed that all naturally occurring determiners are immune to extensional restriction, but note that the unconstrained determiner MANY presented above is not. (Cf., e.g., van Benthem 1983.) Yet, immunity to extensional restriction may serve to exclude from the cube the two most uncommon determiners that we have been considering so far: UNLESS and ELSE. Removing them, the remaining six minimal logical determiners, or combinators, constitute a prism of oppositions. ${ }^{24}$

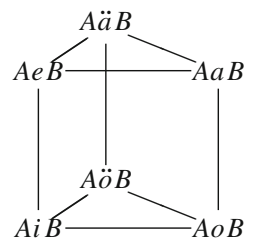

The combinators in the prism are of course mutually related like they are in the cube; more interestingly, like the cube, the prism is contradiction- and converse-complete. This is to say that the validity of syllogistic schemes in all figures can be derived (by conversion) from the validity of those in one figure only, and that the validity of particular ones can be derived (by contraposition) from the validity of general ones, and vice versa. The syllogistics of the prism thus is logically well-behaved, as one may also conclude from the table at the end of this paper.

Immunity of a logical determiner $D$ to extensional restriction implies that $D(A, B)$ only depends on the cardinalities of $A \backslash B, A \cap B$ and $B \backslash A$, and that it can be rendered as a set of triples (not pairs) of natural numbers. Amplifying the method from (van Benthem 1983), a logical determiner can then be represented as a valuation pattern over-not a tree-but this time a pyramid of numbers. If we let mno stand for the

\footnotetext{
24 Independent from linguistic motivations Kraszewski 1956 has singled out precisely this set of categorial combinators. I thank Janusz Ciuciura for pointing out the reference to me.
} 
cardinalities of $A \backslash B(m)$, of $A \cap B(n)$ and of $B \backslash A(o)$, and use $x^{\prime}$ for $x+1$, then the number pyramid can be displayed as indicated below, with next to it the distinctive patterns of three of the minimal logical determiners from the prism. (In the table at the end of this paper larger sections of the various pyramids are displayed.)

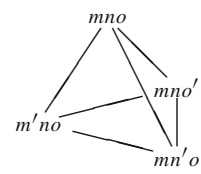

The possibility to present logical determiners this way indicates that computational results obtained in terms of semantic automata running over trees of numbers, can be preserved, in so far as the corresponding automata can be run over pyramids of numbers. (van Benthem 1987)

We have already mentioned the adverbial expressions "never", "always", "sometimes" and "not always" and it seems to be common wisdom that these can also figure binary operators, which can be explicitly restricted by when- or if-clauses. But so can "only" and "not only". We thus find six elementary ways in which we can quantify over a domain of temporal instances or situations, as exemplified by the scheme in (63), with instances in (64).

(63) $A D V S$ if/when $S^{\prime}$.

(64) Don \{never/always/only/sometimes/not always/not only\} talks nonsense when he is drunk.

Assuming a domain of moments, situations, or states as the domain of quantification, the sentences in (64) can be used to express the propositions that $N O, A L L$, ONLY,SOME,NOT_ALL,NOT_ONLY drunk states of Don's are states in which he talks nonsense. ${ }^{25}$ Using "always", there is the reading that on all occasions on which he is drunk, Don talks nonsense; with "only", it is said that if he talks nonsense, he is drunk. In other words, also in the adverbial domain we find precisely the six possibilities from the prism, not the square, of opposition. ${ }^{26}$

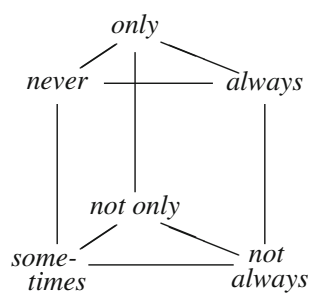

We already observed that "only" does not figure as a unary temporal adverb, while "always", "never" etc. do. One of the reviewers suggests that this may constitute

\footnotetext{
25 Sentences like these notoriously allow for a variety of alternative interpretations, partly correlating with the choice of material that can be taken to be presupposed, that goes in the restrictor, and that goes in the nuclear scope of the quantifying adverb (see, e.g., Hendriks and de Hoop 2001).

26 The apparently compound combinators "not always" and "not only" are again taken as a unit, since here as well "not never" and "not sometimes" are indeed awkward. I take this to provide motivation for taking the two compounds as quasi-compounds.
} 
another argument for not taking "only" to be a determiner. Unlike the other determiners, it does not allow its first argument to be deleted. But the explanation that I favor, instead, builds precisely on the determiner interpretation of "only". Intuitively, the first, focal, argument of "only" involves the specification of a necessary condition for its second argument to obtain, whence its first argument is essential. Indeed, "only" can then make for an essentially temporal reading only in combination with a restrictive clause, temporal or other. Yet, its interpretation seems to be intuitively clear then. Consider the following example.

(65) Raj talks to women only after a couple of beers.

One may quarrel whether it is times, or situations, or cases, ..., that such a sentence can be used to quantify over, but it seems clear that "only" here specifies a necessary (not: sufficient) condition for a certain type of situation to obtain. For a situation to be a "Raj talks to women"-situation it is required, or said to be required, to be a "having had a couple of beers"-situation. Situations of the first kind must be situations of the second kind, which, extensionally, boils down to stating that the set of situations instantiating the latter type includes the set instantiating the first. This is exactly what $O N L Y$ requires. Upon reflection, it is not at all that clear, how such a reading, most prominent I think, should be obtained if, as alternatively suggested, "only" would merely contribute the exclusion of focal alternatives.

A Fork of Oppositions It has been taken a striking point about the original square of opposition that one of its corners, NOT_ALL, is never seen to be realized as a lexical item, neither is NOT_ALWAYS. This has become known as the $o$-problem. As soon as we do no longer conceive of the square as sacrosanct, however, it is also no longer clear why this should be a problem. Moreover, we can also take the fact to heart that ONLY does get lexically realized. ${ }^{27}$ Another distinguished quartet of combinators thus shows up: NO, SOME, ALL and $O N L Y(e, i, a, \ddot{a})$. These four form a fork of oppositions.

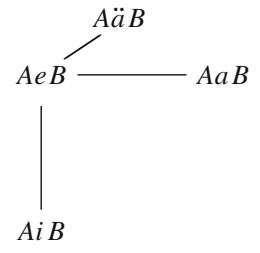

It does not look like these four combinators share a characteristic semantic property, but they are obviously intrinsically related. The whole can be seen to emerge by the three possible types of negation from the junction $A e B$. (As can be seen from the picture, the fork extends in the three dimension of the cube.) The set of four combinators has less expressive power than the cube or the square, but it is converse-complete. The combinators $a$ and $\ddot{a}$ are each others converses, and $e$ and $i$ are self-converse. The syllogistics of the fork, hence, is minimal but transparent. (An overview of the first figure validities in the various systems is given at the end of this paper.)

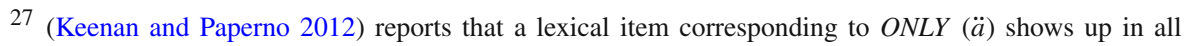
languages in a recent cross-linguistic survey.
} 
Interestingly, we can also observe a fork in the domain of deontic modalities. Neglecting several, in particular performative, aspects of these modalities, we can conceive of them as specifying conditions for some normative or practical goal. They may specify certain possible actions or facts as consistent or inconsistent with the goal ( $i$ and $e$, respectively) or as a necessary or sufficient condition for it ( $\ddot{a}$ and $a$ ). Such conditions can be supplied by elementary modalities "may", "may not", "must" and "should". ${ }^{28}$ For the goal of meeting certain goals or requirements, we may say each of the following. (In the associated categorial proposition $P$ is short for "playing" and $R$ for "meeting the requirements".)

(66) Don $\{$ may / may not / must / should $\}$ play. (PeR, PiR, PäR, PaR.)

That is, playing is stated to be some ( $i$, "may"), or no ( $e$, "may not") way to meet the requirements, or playing is stated a necessary ( $\ddot{a}$, "must") or sufficient ( $a$, "should") for meeting the requirements. Taken thus, the four modalities can also be displayed in the format of a fork.

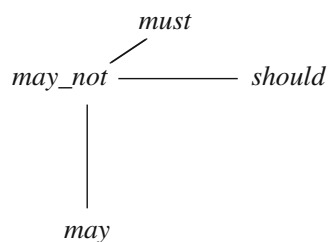

This is all very tentative and sketchy, of course. Still the range of uses of the modalities can be matched with these interpretations. That is, "may (not) $P$ " expresses (in)consistency, and hence indicates or instigates permission (prohibition). "Must $P$ " expresses inevitability of $P$ and is, thus, imperative. (It is taken to say that only if $P$ will things be alright; $P$ is mandatory.) "Should $P$ " proposes that $P$ brings about the goal, and with the qualification "all else being equal", may properly count as advice.

A Diamond The two sections of the cube that we have seen so far both exclude the $x$ and $y$ corners, which indeed look somewhat exotic in the predicative domain, nominal as well as verbal. Surprisingly, perhaps, they are arguably there in the sentential or propositional domain. If we associate the (material) implication with the $a$-corner, then standard propositional logic equivalences give us $i$ for conjunction, and its dual $x$ for disjunction. ${ }^{29}$ Inverse implication would be $\ddot{a}$, of course, and we find $\ddot{a}=\uparrow$, the Sheffer stroke, and $y=\downarrow$, the Quine dagger. A cube of propositional combinators thus can be displayed as follows.

\footnotetext{
28 I have taken "may not" as a quasi-compound expression for exclusion or prohibition, that is, as the denial or negation of "may". I believe the default interpretation of "You may not enter" is that it is not allowed or permitted to enter; not that it is allowed to not enter.

29 We can conceive of sentences as zero-place predicates, which either have the empty tuple (the true) in their denotation, or not, in which case they are false. A conjunction $(P \wedge Q)$, rendered as $P i Q$, then comes out true if and only if the intersection of $P$ and $Q$ is not empty, i.e., if and only if the true is in both $P$ and $Q$, as desired. The three forms of negation can then be uniformly conceived of as complementation, and the cube unfolds as shown.
} 


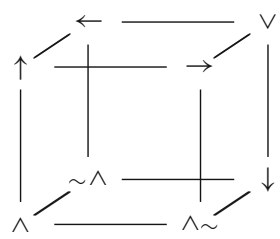

It does not seem to be outrageous to suppose that "and", "or", "if" and "only if" (or postpositional "if") figure as elementary sentential connectives in natural language, and in some rudimentary sense they can be taken to correspond to $\wedge, \vee, \rightarrow$ and $\leftarrow$, respectively. This would give us the following subsection of the cube, in the shape of a (crooked) diamond.

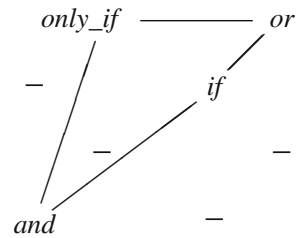

This subsection is, also surprisingly perhaps, converse complete, but negation free. The syllogistics of the diamond is, thus, transparent again, but very minimal (See the table at the end of the paper). Notice that with (propositional) negation added, the full cube gets restored. ${ }^{30}$

In the figure above conjunction and disjunction appear in the two corners of the cube which are positive in both of their arguments, which is surely appropriate. Conjunction ("and") however, is rendered particular, and disjunction ("or") general. It should be noticed that this does by no means conflict with the 'universal' character of "and", and the 'existential' character of "or", because that aspect is of a different nature. It may also be noticed that the choice between particular or general is immaterial if we consider propositions as zero-place predicates. (See footnote above.) The domain that sentences are predicates of has only one member, the true, so a sentence has some truth if and only if it has every truth. Once we start making distinctions among the true, for instance, when we introduce proofs or states of affairs as truth makers, then the choice between particular and general does start making a difference. It seems to me that it is more appropriate, then, to have a conjunction $(P \wedge Q)$ require some proof to be a proof and $P$ and $Q$, rather then every. However, I must submit that I have no substantial opinion on that matter.

\footnotetext{
30 In the last two figures conjunction, and "and", are associated with the $i$-corner of the cube. As a reviewer points out conjunction has a close and natural tie with the universal quantifier, and disjunction with the existential one. For this reason the two have been traditionally associated with the $a$ - and $i$-corners of the cube, respectively. The two connectives plus "nand" $(\uparrow)$ and "nor" $(\downarrow)$, can be seen to form their own (Boolean) square, which also can be cubed, as recently expanded upon in (Westerståhl 2012). However, Westerståhl's Boolean square of dualities does not match the Aristotelean one, in which the $a$-corner is notoriously not symmetric, and $\rightarrow$ comes much closer to $a$ than $\wedge$ does. Westerståhl's findings are therefore orthogonal to the ones discussed in this paper.
} 


\section{Conclusion}

In this paper I have first considered the square of opposition as constituting just one side of a logical cube of opposition. In line with Reichenbach's observations of the cube I have shown its syllogistics to be totally transparent, if not trivial.

By extending our restricted focus on the square, or by more generally lifting a conservativity constraint, we can also, so I claim, open up our eyes to a wider range of structural properties of natural language determiners. More particularly the concept of what is called allergy to nominal restriction has shown relevant for a characterization of the determiners that oppose nominal deletion, and allergy to verbal restriction characterizes those that oppose existential-there and relational-have contexts, and allow non-extensional readings as arguments of intensional transitive verbs. Certain proportional readings of "many" become almost automatically available. These readings have been acknowledged before, but have not been that easily accounted for. Also various contextually readings of "only" become available, on the basis of the simple and I claim intuitive interpretation of it as $\operatorname{ONLY}(\ddot{a})$. In the final Sect. 2 have demonstrated that the square need not figure as the only interesting subsection of the cube, and that others can be detected.

The observations in this paper could not have been stated, not even made, if we were to were to use the square as the sole paradigm, or conservativity as a linguistic universal. If the set of combinators or of determiners is not restricted to begin with, you see more of them.

Open Access This article is distributed under the terms of the Creative Commons Attribution License which permits any use, distribution, and reproduction in any medium, provided the original author(s) and the source are credited.

\section{Appendix}

Valid Syllogistic Schemes of the First Figure

\begin{tabular}{|l|cc|ccccc|}
\hline Name & Syll. & (Reord.) & Cube & Square & Prism & Fork & Diamond \\
\hline Barbara & aaa-1 & äää-1 & $+(+)$ & $+(-)$ & $+(+)$ & $+(+)$ & $+(+)$ \\
Celarent & eae-1 & äee-1 & $+(+)$ & $+(-)$ & $+(+)$ & $+(+)$ & \\
Gaxx & axx-1 & xäx-1 & $+(+)$ & & & & $+(+)$ \\
Xena & xea-1 & exä-1 & $+(+)$ & & & & \\
\hline Borbäro & oäo-1 & aöö-1 & $+(+)$ & & $+(+)$ & & \\
Bärboro & äoo-1 & öaö-1 & $+(+)$ & & $+(+)$ & & \\
Diräi & iäi-1 & aii-1 & $+(+)$ & $-(+)$ & $+(+)$ & $+(+)$ & $+(+)$ \\
Ferio & eio-1 & ieö-1 & $+(+)$ & $+(-)$ & $+(+)$ & & \\
Gyxo & yxo-1 & xyö-1 & $+(+)$ & & & & \\
Gämyny & äyy-1 & yay-1 & $+(+)$ & & & & \\
Omeny & oey-1 & eöy-1 & $+(+)$ & & & & \\
Xodis & xoi-1 & öxi-1 & $+(+)$ & & & & \\
\hline \multicolumn{7}{|l}{ Other figures } \\
\hline \multicolumn{70}{|c|}{} \\
\hline
\end{tabular}




\section{Six Pyramids of Numbers}

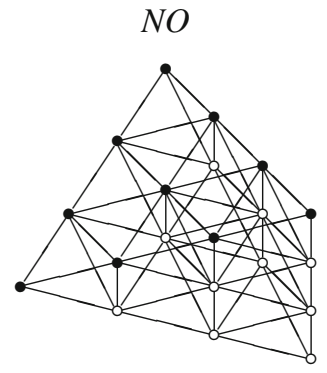

SOME

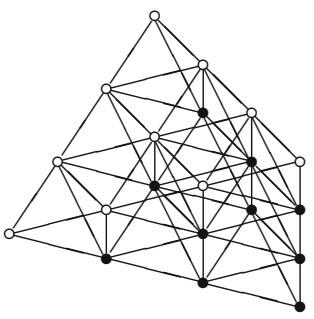

ALL

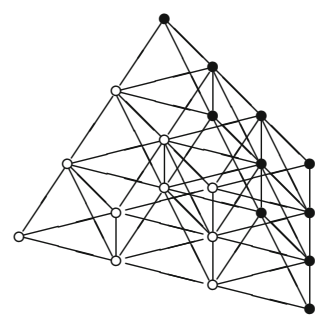

NOT_ALL

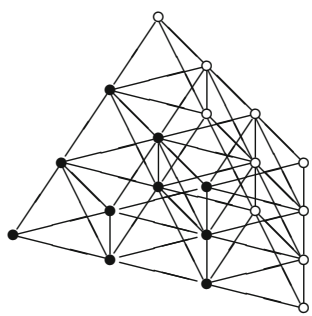

ONLY

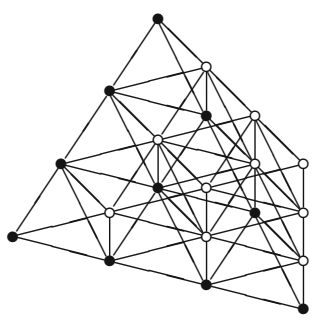

NOT_ONLY

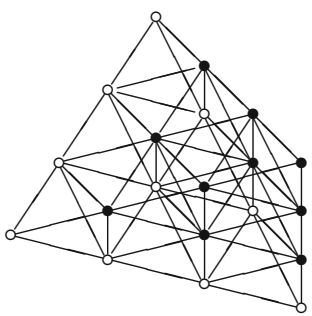

\section{References}

Aristotle. (1984). De interpretatione. In J. Barnes (Ed.) The complete works of aristotle, revised oxford translation, Princeton UP. pp. 25-38. Translation by Ackrill, J. L.; electronic edition: InteLex Past Masters, http://pm.nlx.com/.

Barwise, J., \& Cooper, R. (1981). Generalized quantifiers in natural language. Linguistics and Philosophy, $4(1), 159-220$.

Bastiaanse, H. (2014). The intensional many-conservativity reclaimed. Journal of Philosophical Logic, 43(5), 883-901.

Chierchia, G. (1992). Anaphora and dynamic binding. Linguistics and Philosophy, 15(2), 111-183.

Cohen, A. (2001). Relative readings of many, often and generics. Natural Language Semantics, 9, 41-67. de Mey, S. (1991). 'Only' as a determiner and as a generalized quantifier. Journal of Semantics, 8(1), 91-106.

de Mey, S. (1996). Generalized quantifier theory and the semantics. In J. van der Does \& J. van Eijck (Eds.), Quantifiers, logic, and language (pp. 269-279). Stanford: CSLI Publications.

de Morgan, A. (1846). On the structure of the syllogism. Transactions of the Cambridge Philosophical Society, 8, pp. 159-220. Reprinted in on the Syllogism, ed. Peter Heath, Routledge and Kegan, London (1966).

Dekker, P. (2001). On if and only. In R. Hastings, B. Jackson, \& Z. Zvolenszky (Eds.), Proceedings from SALT XI (pp. 114-133). Ithaca, NY: CLC Publications.

Fernando, T., \& Kamp, H. (1996). Expecting many. In T. Galloway \& J. Spence (Eds.), Proceedings from SALT VI (pp. 53-68). Cornell.

Francez, N., \& Ben-Avi, G. (2014). Proof-theoretic reconstruction of generalized quantifiers. Journal of Semantics, 31, 1-59.

Greer, K. A. (2014). Extensionality and natural language quantification: the case of many and few. Linguistics and Philosophy, 37(4), 315-351.

Hendriks, P., \& de Hoop, H. (2001). Optimality theoretic semantics. Linguistics and Philosophy, 24(1), $1-32$.

Herburger, E. (1997). Focus and weak noun phrases. Natural Language Semantics, 5, 53-78.

Hunter, T., \& Lidz, J. (2013). Conservativity and learnability of determiners. Journal of Semantics, 30(3), $315-334$. 
Johnsen, L. (1987). There-sentences and generalized quantifiers. In P. Gärdenfors (Ed.), Generalized quantifiers (pp. 93-107). Dordrecht: Reidel.

Kanazawa, M. (1994). Weak vs strong readings of donkey sentences and monotonicity inference in a dynamic setting. Linguistics and Philosophy, 17(2), 109-148.

Keenan, E. L. (1987). A semantic definition of “indefinite NP”. In E. J. Reuland \& A. G. B. ter Meulen (Eds.), The representation of (in)definiteness (pp. 286-317). Cambridge, MA: MIT Press.

Keenan, E. L. (1996). The semantics of determiners. In S. Lappin (Ed.), Handbook of contemporary semantic theory language (pp. 41-63). Oxford: Blackwell.

Keenan, E. L. (2003). The definiteness effect: Semantics or pragmatics? Natural Language Semantics, 11, $187-216$.

Keenan, E. L., \& Paperno, D. (2012). Handbook of quantifiers in natural language. Dordrecht: Springer.

Kraszewski, Z. (1956). Logika stosunków zakresowych (rachunek zdań zakresowych). Studia Logica, 4(1), 63-116.

Krifka, M. (1993). Focus and presupposition in dynamic interpretation. Journal of Semantics, 10(4), 269300.

Moltmann, F. (2013). Abstract objects and the semantics of natural language. Oxford: Oxford University Press.

Peirce, C. S. (1880). On the Algebra of logic. In C. Hartshorne \& P. Weiss (eds.) The collected papers of Charles Sanders Peirce, III, exact logic, Cambridge: Harvard UP. §173-181. Electronic edition: InteLex Past Masters http://pm.nlx.com/.

Peters, S., \& Westerståhl, D. (2006). Quantifiers in language and logic. Oxford: Clarendon Press.

Reichenbach, H. (1952). The syllogism revised. Philosophy of Science, 19(1), 1-16.

Rooth, M. (1992). A theory of focus interpretation. Natural Language Semantics, 1(1), 75-116.

van Benthem, J. (1983). Determiners and logic. Linguistics and Philosophy, 6, 447-478.

van Benthem, J. (1987). Semantic automata. In J. Groenendijk, D. de Jongh, \& M. Stokhof (Eds.), Studies in discourse representation theory and the theory of generalized quantifiers (pp. 1-25). Dordrecht: Foris.

van Benthem, J. (1991). Linguistic universals in logical semantics. In D. Zaefferer (Ed.), Semantic universals and universal semantics (pp. 17-36). Berlin: Foris.

van Eijck, J., \& Kamp, H. (1997). Representing discourse in context. In J. van Benthem \& A. ter Meulen (Eds.), Handbook of logic and language (pp. 179-237). Amsterdam: Elsevier.

van Rooij, R., \& Schulz, K. (2007). Only: Meaning and implicatures. In M. Aloni, A. Butler, \& P. Dekker (Eds.), Questions in dynamic semantics, CRiSPI 17 (pp. 193-223). Amsterdam: Elsevier.

von Fintel, K. (1994). Restrictions on quantifier domains. Ph.D. thesis, University of Massachusetts, Amherst.

von Fintel, K., \& Matthewson, L. (2008). Universals in semantics. The Linguistic Review, 25(1), 139-201.

Westerståhl, D. (2014). Generalized quantifiers. In E. N. Zalta (Ed.) The stanford encyclopedia of philosophy, http://plato.stanford.edu/archives/spr2014/entries/generalized-quantifiers/. Spring 2014 edition.

Westerståhl, D. (2012). Traditional vs. modern squares of opposition, and beyond. In J. Y. Beziau \& G. Payette (Eds.), The square of opposition: A general framework for cognition (pp. 195-229). Berlin: Peter Lang.

Zuber, R. (2004). A class of non-conservative determiners in Polish. Linguisticae Investigationes, 27, 147165. 